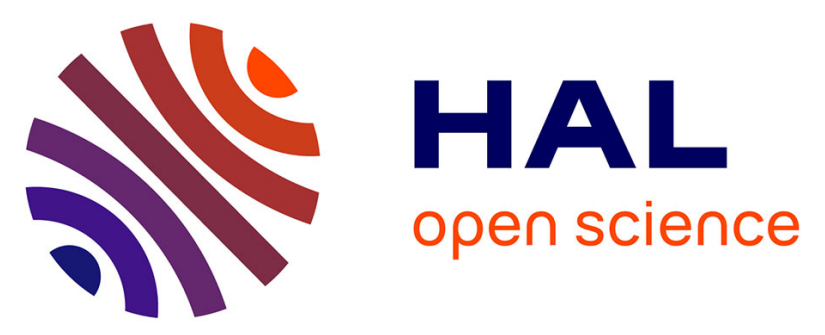

\title{
Weakly nonlinear analysis of Rayleigh-Bénard convection in a non-Newtonian fluid between plates of finite conductivity: Influence of shear-thinning effects
}

Mondher Bouteraa, Chérif Nouar

\section{- To cite this version:}

Mondher Bouteraa, Chérif Nouar. Weakly nonlinear analysis of Rayleigh-Bénard convection in a nonNewtonian fluid between plates of finite conductivity: Influence of shear-thinning effects. Physical Review E , 2016, 10.1103/PhysRevE.92.063017 . hal-02447344

\author{
HAL Id: hal-02447344 \\ https://hal.science/hal-02447344
}

Submitted on 21 Jan 2020

HAL is a multi-disciplinary open access archive for the deposit and dissemination of scientific research documents, whether they are published or not. The documents may come from teaching and research institutions in France or abroad, or from public or private research centers.
L'archive ouverte pluridisciplinaire HAL, est destinée au dépôt et à la diffusion de documents scientifiques de niveau recherche, publiés ou non, émanant des établissements d'enseignement et de recherche français ou étrangers, des laboratoires publics ou privés. 


\title{
Weakly nonloinear analysis of Rayleigh-Bénard convection in a non-Newtonian fluid between plates of finite conductivity: Influence of shear-thinning effects
}

\author{
Mondher Bouteraa and Chérif Nouar* \\ LEMTA UMR 7563 CNRS - Université de Lorraine \\ ENSEM, 2 Avenue de la Forêt de Haye, \\ TSA 60604 - 54516 Vandoeuvre lès Nancy cedex, France
}

(Dated: October 3, 2015)

\begin{abstract}
Finite-amplitude thermal convection in a shear-thinning fluid layer between two horizontal plates of finite thermal conductivity is considered. Weakly nonlinear analysis is adopted as a first approach to investigate non linear effects. The rheological behavior of the fluid is described by the Carreau model. As a first step, the critical conditions for the onset of convection are computed as a function of the ratio $\xi$ of the thermal conductivity of the plates to the thermal conductivity of the fluid. In agreement with the literature, the critical Rayleigh number $R a_{c}$ and the critical wave number $k_{c}$ decrease from 1708 to 720 and from 3.11 to 0 , when $\xi$ decreases from infinity to zero. In the second step, the critical value $\alpha_{c}$ of the shear-thinning degree above which the bifurcation becomes subcritical is determined. It is shown that $\alpha_{c}$ increases with decreasing $\xi$. The stability of rolls and squares is then investigated as a function of $\xi$ and the rheological parameters. The limit value $\xi_{c}$, below which squares are stable, decreases with increasing shear-thinning effects. This is related to the fact that shear-thinning effects increase the nonlinear interactions between sets of rolls that constitute the square patterns [3]. For a significant deviation from the critical conditions, nonlinear convection terms and nonlinear viscous terms become stronger leading to a further diminution of $\xi_{c}$. The dependency of the heat transfer on $\xi$ and the rheological parameters is reported. It is consistent with the maximum heat transfer principle. Finally, the flow structure and the viscosity field are represented for weakly and highly conducting plates.
\end{abstract}

Keywords: bifurcation, Rayleigh-Bénard convection, shear-thinning fluid

*cherif.nouar@univ-lorraine.fr 


\section{INTRODUCTION}

Recently, a weakly nonlinear stability analysis of thermal convection in a layer of a non-Newtonian fluid between two horizontal plates heated from below was considered by Bouteraa et al. [3]. Assuming the fluid purely viscous and shear-thinning, Bouteraa et al. [3] studied the influence of shear-thinning effects on the nature of the bifurcation, the convection intensity and the pattern selection. The possibility of wall slip was taken into account by using Navier's slip law at top and bottom walls. It was shown that the bifurcation is supercritical for moderately shear-thinning effects and becomes subcritical for strongly shear-thinning effects. The critical value of the degree of shear-thinning $\alpha_{c}$ defined by Eq. (14), above which the bifurcation becomes subcritical is determined as a function of a dimensionless slip length parameter. Bouteraa et al. [3] demonstrated that near the threshold of the convection, only rolls are stable and this stability is reinforced by the shear-thinning behavior. It is shown that shear-thinning effects increase the interaction between sets of rolls that constitute square and hexagon patterns. In the above analysis, it was assumed that the plates are of infinite thermal conductivity $\hat{k}_{p}$ and heat capacity.

In experimental situations, however, the thermal conductivity of the plates is finite. Furthermore, in some situations, the plates are much poorer conductors than the fluid. For instance, in LeGal \& Croquette [21], the plates are made of Plexiglas and the fluid is water. The ratio $\xi$ of the thermal conductivity of the plates $\hat{k}_{p}$ to the thermal conductivity of the fluid $\hat{k}$ is $\xi=0.4$. In Kebiche [18] and Kebiche et al. [19], the horizontal plates are made of Polycarbonate and the fluid is an aqueous solution of CarboxyMethylCellulose or Carbopol, the ratio $\xi$ is estimated to $\xi=0.25$. In these situations, as well as those encountered in some engineering convection problems and in geophysics, the assumption that the plates are held at fixed and uniform temperatures loses its validity. Indeed, temperature disturbance penetrates the wall. More general boundary conditions have to be used. The temperature and the heat flux obey a continuity equation at the boundaries.

For a Newtonian fluid, the effect of imperfect conducting plates on the Rayleigh-Bénard convection was investigated by several authors. First, in the linear theory frame, Sparrow et al. [32] and Hurle et al. [16] (see also Riahi [30], Clever \& Busse [8] and Holmedal 
et al. [15] for plates of arbitrary thicknesses and conductivities) showed that the critical Rayleigh number $R a_{c}$ and the critical wave number $k_{c}$, vary continuously from 1708 to 720 and from 3.11 to 0 , when the ratio of thermal conductivities decreases from infinity to zero. According to Cerisier et al. [6] a temperature fluctuation occurring in the liquid close to a nearly insulating plate persists and distorts the temperature distribution. This distribution can led to instability of the fluid layer. As a consequence, the temperature gradient is small and the fluid organizes in a pattern with a small wavenumber.

Exploiting the fact that for nearly insulating walls $(\xi<<1)$, the horizontal scale of convection is much larger than the depth of the fluid, Busse \& Riahi [4] considered weakly nonlinear three-dimensional solutions in the case of infinitely thick plates. They showed that near the onset, square convection cells are the stable planform, in contrast with two-dimensional rolls which are the only stable convection pattern in a symmetrical situation with isothermal boundary conditions. This result was confirmed and extended to fully nonlinear convection by Proctor [28] using a "shallow water theory" adapted for the Rayleigh-Bénard convection by Chapman \& Proctor [7]. The thickness of the plates was assumed finite. Jenkins \& Proctor [17] considered three-dimensional finite-amplitude thermal convection with finite thickness and finite thermal conductivity of the bounding plates. They determined the critical value $\xi_{c}$ of the thermal conductivities ratio at which the preferred planform changes from square cell $\left(\xi<\xi_{c}\right)$ to roll $\left(\xi>\xi_{c}\right)$, as function of the Prandlt number. When the thickness of the plates is of the same order as that of the fluid layer and for $\operatorname{Pr} \geq 10$, rolls should be observed when $\xi>1$ and squares when $\xi<1$. This is in agreement with LeGal \& Croquette's experiments [21].

The objective of the present paper is twofold. Firstly, to determine the critical value of the shear-thinning degree above which the bifurcation becomes subcritical as a function of $\xi$. Secondly, to study the influence of shear-thinning effects on $\xi_{c}$. We hope that our findings will shed new light on the interpretation of the results obtained by Kebiche [18] and Kebiche et al. [19], although the fluid used in [19] is not only shear-thinning but has also a yield stress.

The paper is organized as follows. In section II, the mathematical formulation of the problem is presented. In section III, the linear stability analysis for the onset of convective flow is reinvestigated. The critical Rayleigh number $\left(R a_{c}\right)$ and wavenumber $\left(k_{c}\right)$ are 
determined as a function of $\xi$. Section IV presents briefly the procedure used in the weakly nonlinear stability analysis. The results are discussed in section $\mathrm{V}$. The critical value of the shear-thinning degree above which the bifurcation becomes subcritical is determined as a function of $\xi$. The pattern selection near the threshold of convection is investigated in terms of $\alpha$ and $\xi$. For a significant deviation from the critical conditions, higher-order solutions are computed in section VI. Section VII provides information on the flow structure and the heat transfer. A concluding discussion is given in the last section of the paper.

\section{PROBLEM FORMULATION}

\section{A. General equations and parameters}

We consider a layer of shear-thinning fluid of depth $\hat{d}$ confined between two horizontal plates that are infinite in extent and which have a thickness $\Lambda \hat{d}$, where $\Lambda$ is of order unity. The outer surface of the bottom and top plates are kept at constant temperatures respectively $\hat{T}_{0}+\Delta \hat{T} / 2$ and $\hat{T}_{0}-\Delta \hat{T} / 2$, with $\Delta \hat{T}>0$. The fluid has density $\hat{\rho}$, thermal conductivity $\hat{k}$, thermal coefficient expansion (at constant pressure) $\hat{\beta}$ and viscosity $\hat{\mu}_{0}$ at zero shear rate. The thermal conductivity of the slabs is $\hat{k}_{p}$. Because of the thermal expansion, the temperature difference between the two plates, induces a vertical density stratification. Heavy cold fluid is above a light warm fluid. For small $\Delta \hat{T}$, the fluid remains at rest and the heat is transferred by conduction. In the fluid, $0<\hat{z}<\hat{d}$, the hydrostatic solution and the temperature profile are:

$$
\frac{d \hat{P}}{d \hat{z}}=-\hat{\rho}_{0} \hat{g}\left[1-\hat{\beta}\left(\hat{T}-\hat{T}_{0}\right)\right] \quad \text { and } \quad \hat{T}_{\text {cond }}=\hat{T}_{0}+\frac{\Delta \hat{T}}{1+2 \Lambda / \xi}\left[\frac{1}{2}-\frac{\hat{z}}{\hat{d}}\right]
$$

where, $\hat{g}$ is the acceleration due to gravity. Here, the z-axis is directed upwards, with the origin located at the bottom plate. The reference temperature $\hat{T}_{0}$ is the temperature in the middle of the fluid layer and $\hat{\rho}_{0}$ is the fluid density at $\hat{T}_{0}$. The temperature difference between the top and the bottom of the fluid layer is $\Delta \hat{T}_{f}=\Delta \hat{T} /(1+2 \Lambda / \xi)$.

The temperature profile in the top and bottom plates are:

$$
\hat{T}_{\text {cond }}=\hat{T}_{0}+\frac{\Delta \hat{T}}{\xi+2 \Lambda}\left[1-\frac{1}{2} \xi-\frac{\hat{z}}{\hat{d}}\right], \quad \hat{d} \leq \hat{z} \leq(1+\Lambda) \hat{d}
$$


and

$$
\hat{T}_{\text {cond }}=\hat{T}_{0}+\frac{\Delta \hat{T}}{\xi+2 \Lambda}\left[\frac{1}{2} \xi-\frac{\hat{z}}{\hat{d}}\right], \quad-\Lambda \hat{d} \leq \hat{z} \leq 0 .
$$

When $\xi$ is low, a large part of $\Delta \hat{T}$ occurs across the plates, and remains only a small part $\Delta \hat{T}_{f}$ of $\Delta \hat{T}$, acting as the driving force for the convection. When $\Delta T_{f}$, exceeds a critical value, the buoyancy force overcomes, the dissipative effects, i.e., viscous and heat diffusion, the convection sets in and a so-called convective patterns emerge. The stability of the hydrostatic solution is considered by introducing temperature and pressure perturbation as well as a fluid motion. Boussinesq approximation is adopted, i.e., the temperature dependence of the fluid properties can be neglected except for the temperature induced density difference in the buoyant force that drives the flow. The heat production due to viscosity is neglected. Here and in what follows, the quantities with hat (.) are dimensional. Distances are scaled with $\hat{d}$, velocity with $\hat{\kappa} / \hat{d}$, where $\hat{\kappa}$ is the thermal diffusivity of the fluid, time with $\hat{d}^{2} / \hat{\kappa}$ (characteristic time scale of thermal diffusion), temperature with $\Delta \hat{T}_{f}$, pressure and stresses with $\hat{\kappa} \hat{\mu}_{0} / \hat{d}^{2}$. Using these scales, the dimensionless perturbation equations read:

$$
\begin{aligned}
& \boldsymbol{\nabla} \cdot \boldsymbol{u}=0 \\
& \frac{1}{P r}\left[\frac{\partial \boldsymbol{u}}{\partial t}+\boldsymbol{u} \cdot \boldsymbol{\nabla} \boldsymbol{u}\right]=-\boldsymbol{\nabla} p+R a \theta \boldsymbol{e}_{z}+\nabla \cdot \boldsymbol{\tau} \\
& \frac{\partial \theta}{\partial t}+\boldsymbol{u} \cdot \boldsymbol{\nabla} \theta=\boldsymbol{u} \cdot \boldsymbol{e}_{z}+\nabla^{2} \theta
\end{aligned}
$$

in the fluid, and

$$
\frac{\partial \tilde{\theta}}{\partial t}=\frac{\hat{\kappa}_{p}}{\hat{\kappa}} \nabla^{2} \tilde{\theta}
$$

in the bounding slabs. Here, $\boldsymbol{e}_{z}$ denotes the unit vector in the vertical direction, $\boldsymbol{u}(\boldsymbol{x}, t)=$ $u \boldsymbol{e}_{x}+v \boldsymbol{e}_{y}+w \boldsymbol{e}_{z}$ is the fluid velocity and $p(\boldsymbol{x}, t)$ and $\theta(\boldsymbol{x}, t)$ represent the pressure and temperature deviations from their values in the conduction state. The temperature perturbation in the slabs is denoted $\tilde{\theta}(\boldsymbol{x}, t)$. The position vector $\boldsymbol{x}$ has components $x, y, z$. The Rayleigh number $R a$ and the Prandtl number $\operatorname{Pr}$ are defined by :

$$
R a=\frac{\hat{\rho}_{0} \hat{g} \hat{\beta} \Delta \hat{T}_{f} \hat{d}^{3}}{\hat{\kappa} \hat{\mu}_{0}} \quad ; \quad \operatorname{Pr}=\frac{\hat{\mu}_{0}}{\hat{\rho}_{0} \hat{\kappa}} .
$$

Generally, for non-Newtonian fluids, $\operatorname{Pr}>>1$, i.e., the viscous diffusion time is shorter than the thermal diffusion time. 


\section{B. Rheological model and parameters}

The fluid is assumed to be purely viscous and shear-thinning. The viscous stress-tensor

$$
\boldsymbol{\tau}=\mu(\Gamma) \dot{\gamma} \quad \text { with } \quad \dot{\gamma}=\boldsymbol{\nabla} \boldsymbol{u}+(\boldsymbol{\nabla} \boldsymbol{u})^{T}
$$

the rate-of-strain tensor, of second invariant

$$
\Gamma=\frac{1}{2} \dot{\gamma}_{i j} \dot{\gamma}_{i j}
$$

The Carreau model is given by

$$
\frac{\hat{\mu}-\hat{\mu}_{\infty}}{\hat{\mu}_{0}-\hat{\mu}_{\infty}}=\left(1+\hat{\lambda}^{2} \hat{\Gamma}\right)^{\frac{n_{c}-1}{2}}
$$

with $\hat{\mu}_{0}$ and $\hat{\mu}_{\infty}$ the viscosities at low and high shear rate, $\left(n_{c}<1\right)$ the shear-thinning index, $\hat{\lambda}$ the characteristic time of the fluid. The location of the transition from the Newtonian plateau to the shear-thinning regime is determined by $\hat{\lambda}$, since $1 / \hat{\lambda}$ defines the characteristic shear rate for the onset of shear-thinning. Increasing $\hat{\lambda}$ reduces the Newtonian plateau to lower shear rates. The infinite shear viscosity, $\hat{\mu}_{\infty}$, is generally associated with a breakdown of the fluid, and is frequently significantly smaller $\left(10^{-3}\right.$ to $10^{-4}$ times smaller $)$ than $\hat{\mu}_{0}$, see Bird et al. [2] and Tanner [34]. The ratio $\hat{\mu}_{\infty} / \hat{\mu}_{0}$ will be thus neglected in the following. The dimensionless effective viscosity is then

$$
\mu=\frac{\hat{\mu}}{\hat{\mu}_{0}}=\left(1+\lambda^{2} \Gamma\right)^{\frac{n_{c}-1}{2}} \quad \text { with } \quad \lambda=\frac{\hat{\lambda}}{\hat{d}^{2} / \hat{\kappa}} .
$$

The Newtonian behavior, $\hat{\mu}=\hat{\mu}_{0}$, is obtained by setting $n_{c}=1$ or $\hat{\lambda}=0$.

For a small amplitude disturbance, the viscosity can be expanded about the hydrostatic solution,

$$
\mu=1+\left(\frac{n_{c}-1}{2}\right) \lambda^{2} \Gamma+\frac{1}{2}\left(\frac{n_{c}-1}{2}\right)\left(\frac{n_{c}-3}{2}\right) \lambda^{4} \Gamma^{2}+\ldots
$$

At lowest nonlinear order, a relevant rheological parameter is the 'degree of shearthinning'

$$
\alpha=\left|\frac{d \mu}{d \Gamma}\right|_{\Gamma=0}=\frac{1-n_{c}}{2} \lambda^{2}
$$




\section{Boundary conditions}

For the perturbation velocity $\boldsymbol{u}$, the conditions imposed on the top and bottom plates are either no-slip (no-slip boundary conditions NSBC), which implies

$$
\boldsymbol{u}=0 \quad \text { at } \quad z=0,1
$$

or stress-free (stress-free boundary conditions SFBC), which implies

$$
\frac{\partial u}{\partial z}=\frac{\partial v}{\partial z}=w=0 \quad \text { at } \quad z=0,1
$$

For thermal boundary conditions, a constant and uniform temperature is assumed on the outer surface of each plate

$$
\tilde{\theta}=0 \quad \text { at } \quad z=-\Lambda, 1+\Lambda
$$

The continuity condition for temperature and heat flux are

$$
\begin{array}{rll}
\theta=\tilde{\theta} \quad \text { at } & z=0,1, \\
D \theta=\xi D \tilde{\theta} \quad \text { at } & z=0,1 .
\end{array}
$$

Where $D \equiv \frac{\partial}{\partial z}$ and $\xi=\frac{\hat{k}_{p}}{\hat{k}}$.

\section{Remark:}

The governing equations (5) and (6) with the constitutive equation (9) and the boundary conditions (15)-(19) are reflection-symmetric about the midplane $z=1 / 2$. The action of this so-called Boussinesq symmetry is

$$
[u, v, w, \theta, p](t, x, y, z) \rightarrow[u, v,-w, \theta, p](t, x, y, 1-z)
$$

\section{Reduction: elimination of the pressure}

In the momentum equations, the pressure field can be eliminated using the curl of Eq. (5). We then take the curl of Eq. (5) one more time. Using the continuity equation, and projecting onto $\boldsymbol{e}_{z}$, we get the following evolution equations for the vertical vorticity $\zeta$ and 
the vertical velocity $w$ :

$$
\begin{aligned}
& \frac{\partial \zeta}{\partial t}+\boldsymbol{e}_{z} \cdot \boldsymbol{\nabla} \times[(\boldsymbol{u} \cdot \boldsymbol{\nabla}) \boldsymbol{u}]=\operatorname{Pr} \Delta \zeta+\operatorname{Pr} \boldsymbol{e}_{z} \cdot \boldsymbol{\nabla} \times[\boldsymbol{\nabla} \cdot(\mu-1) \dot{\gamma}] \\
& \frac{\partial \boldsymbol{\nabla}^{2} w}{\partial t}-\boldsymbol{e}_{z} \cdot[\boldsymbol{\nabla} \times \boldsymbol{\nabla} \times[(\boldsymbol{u} . \boldsymbol{\nabla}) \boldsymbol{u}]]=\operatorname{Pr} \Delta^{2} w+\operatorname{Ra} \operatorname{Pr} \boldsymbol{\nabla}_{H}^{2} \theta- \\
& \operatorname{Pr}[\boldsymbol{\nabla} \times \boldsymbol{\nabla} \times[\boldsymbol{\nabla} \cdot(\mu-1) \dot{\gamma}]] \cdot \boldsymbol{e}_{z}, \\
& \frac{\partial \theta}{\partial t}+(\boldsymbol{u} \cdot \nabla) \theta=w+\nabla^{2} \theta \\
& \frac{\partial \tilde{\theta}}{\partial t}=\frac{\hat{\kappa}_{p}}{\hat{\kappa}} \nabla^{2} \tilde{\theta}
\end{aligned}
$$

where

$$
\zeta=\frac{\partial v}{\partial x}-\frac{\partial u}{\partial y} \quad \text { and } \quad \nabla_{H}^{2}=\frac{\partial^{2}}{\partial x^{2}}+\frac{\partial^{2}}{\partial y^{2}}
$$

From the continuity equation and the vertical vorticity definition, one can deduce the horizontal velocity components $(u, v)$ :

$$
\boldsymbol{\nabla}_{H}^{2} u=-\frac{\partial^{2} w}{\partial x \partial z}-\frac{\partial \zeta}{\partial y} \quad ; \quad \nabla_{H}^{2} v=-\frac{\partial^{2} w}{\partial y \partial z}+\frac{\partial \zeta}{\partial x}
$$

The boundary conditions for $w$ are:

$$
w=D w=0 \quad \text { at } \quad z=0,1 \quad \text { for NSBC, }
$$

and

$$
w=D^{2} w=0 \quad \text { at } \quad z=0,1 \quad \text { for SFBC } .
$$

For the temperature, the boundary conditions are :

$$
\begin{array}{lll}
\tilde{\theta}=0 & \text { at } & z=-\Lambda, 1+\Lambda, \\
\theta=\tilde{\theta} & \text { at } & z=0,1, \\
D \theta=\xi D \tilde{\theta} & \text { at } & z=0,1 .
\end{array}
$$

In the following, as in Chapman \& Proctor [7], Proctor [28] and Carriere et al. [5], we will assume that $\hat{\kappa}_{p} / \hat{\kappa}=\xi$. 


\section{LINER STABILITY ANALYSIS}

\section{A. Critical conditions and critical modes}

In the linear theory, $\boldsymbol{u}$ and $\theta$ are assumed infinitesimal. The nonlinear terms in (20)-(22) can be neglected. We obtain :

$$
\begin{aligned}
& \frac{1}{\operatorname{Pr}} \frac{\partial \zeta}{\partial t}=\Delta \zeta, \\
& \frac{1}{\operatorname{Pr}} \frac{\partial \Delta w}{\partial t}=\Delta^{2} w+R a \Delta_{H} \theta, \\
& \frac{\partial \theta}{\partial t}=w+\Delta \theta, \\
& \frac{\partial \tilde{\theta}}{\partial t}=\frac{\hat{\kappa}_{p}}{\hat{\kappa}} \Delta \tilde{\theta} .
\end{aligned}
$$

At this stage, no non-Newtonian effects enter the problem. The vertical vorticity decouples and obeys a diffusion equation and thus can be ignored in the linear theory. For equations (31)-(33), we seek a normal mode solution

$$
\left[\begin{array}{c}
w(x, y, z, t) \\
\theta(x, y, z, t) \\
\tilde{\theta}(x, y, z, t)
\end{array}\right]=\left[\begin{array}{c}
F_{11}(z) \\
G_{11}(z) \\
\tilde{G}_{11}(z)
\end{array}\right] f(x, y) \exp (s t),
$$

where $s=s_{r}+i s_{i}$, is a complex number and $f(x, y)$ satisfies the two-dimensional Helmoltz equation $\Delta_{H} f=-k^{2} f$. Here $k$ is the norm of the horizontal wavenumber $\boldsymbol{k}$. Substituting (34) into (31)-(33), leads to the differential equations

$$
\begin{aligned}
& s \operatorname{Pr}^{-1}\left(D^{2}-k^{2}\right) F_{11}=-k^{2} R a G_{11}+\left(D^{2}-k^{2}\right)^{2} F_{11}, \\
& s G_{11}=F_{11}+\left(D^{2}-k^{2}\right) G_{11}, \\
& s \tilde{G}_{11}=\frac{\hat{\kappa}_{p}}{\hat{\kappa}}\left(D^{2}-k^{2}\right) \tilde{G}_{11} .
\end{aligned}
$$

It may be shown easily that $s$ is real. The boundary conditions are:

$$
\begin{array}{lll}
F_{11}=D F_{11}=0 & \text { at } & z=0,1 \quad \text { For NSBC } \\
F_{11}=D^{2} F_{11}=0 & \text { at } & z=0,1 \quad \text { For SFBC } \\
\tilde{G}_{11}=0 & \text { at } & z=-\Lambda, 1+\Lambda, \\
G_{11}=\tilde{G}_{11} & \text { at } & z=0,1 \\
D G_{11}=\xi D \tilde{G}_{11} & \text { at } & z=0,1
\end{array}
$$


The set of differential equations (35)-(37) is an eigenvalue problem where $s$ is the eigenvalue and $\boldsymbol{X}_{11}=\left(F_{11}, G_{11}, \tilde{G}_{11}\right)$ the eigenvector. It can be written

$$
s \boldsymbol{M} \cdot \boldsymbol{X}_{11}=\boldsymbol{L} \cdot \boldsymbol{X}_{11} .
$$

Actually, Eq. (37) can be solved analytically:

$$
\begin{aligned}
& \tilde{G}_{11}(z)=G_{11}(z=1) \frac{\sinh (\tilde{k}(1+\Lambda-z))}{\sinh (\tilde{k} \Lambda)} \quad ; \quad 1 \leq z \leq 1+\Lambda, \\
& \tilde{G}_{11}(z)=G_{11}(z=0) \frac{\sinh (\tilde{k}(\Lambda+z))}{\sinh (\tilde{k} \Lambda)} \quad ; \quad-\Lambda \leq z \leq 0,
\end{aligned}
$$

with $\tilde{k}=\sqrt{k^{2}+s \hat{\kappa} / \hat{\kappa}_{p}}$. Hence, the eigenvalue problem (35)-(37) can be restrained to the fluid domain, i.e. Eqs (35) - (36), with the boundary conditions

$$
D G_{11}= \pm \xi \tilde{k} G_{11} \operatorname{coth} \tilde{k} \Lambda \quad ; \quad z=0,1
$$

The eigenvalue problem (35)-(36) with the boundary conditions (46) is solved using a Chebyshev collocation method. The functions, $F_{11}$ and $G_{11}$ are expanded in series of Chebyshev polynomial series of order $N$. The $2(N+1)$ unknowns are determined at the Gauss-Lobatto nodes

$$
z_{j}=\frac{1}{2}\left(\cos \left(\frac{\pi j}{N}\right)+1\right) \quad j=0,1, \ldots, N
$$

Since $\tilde{k}$ depends on $s$, an iterative process is implemented. The eigenvector $\boldsymbol{X}_{11}$ is normalized such that

$$
G_{11}(z=1 / 2)=1 \text {. }
$$

The marginal stability curve $R a(k)$ is determined by the condition $s=0$. The minimum of the marginality stability curve gives the critical Rayleigh number $R a_{c}$ and $k_{c}$ respectively. Figure ?? displays the variation of $R a_{c}$ and $k_{c}$ as a function of the ratio $\xi$ of the thermal conductivity of the boundary to that of the fluid in the case of NSBC and SFBC. The dimensionless thickness of the plates is fixed at $\Lambda=1$. These results are in very good quantitative agreement with those obtained by Sparrow et al. [32], Proctor[28], Jenkins and Proctor [17], Carriere et al. [5] and Cerisier et al. [6]. The wavelength of the convection becomes larger with decreasing $\xi$ and the critical Rayleigh number $R a_{c}$ is also reduced. 


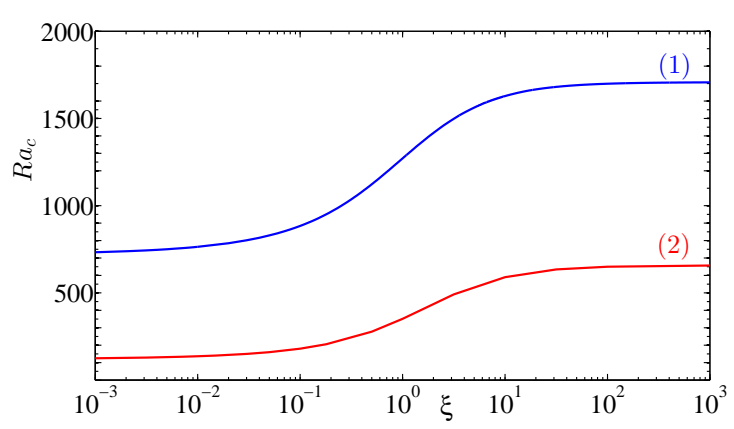

(a)

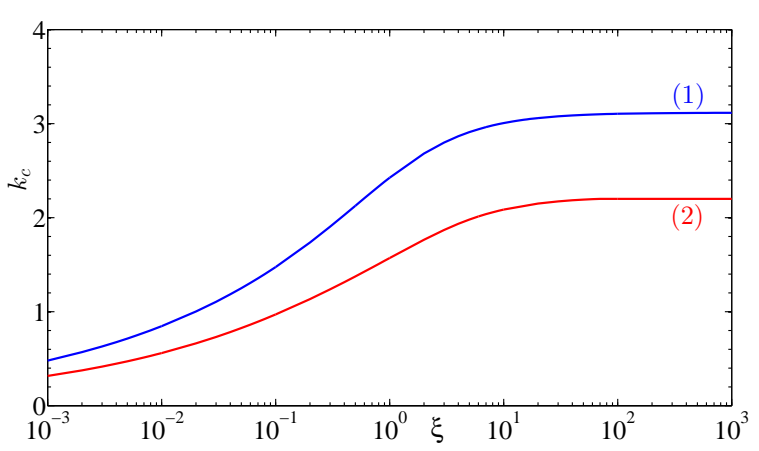

(b)

FIG. 1. Critical Rayleigh number (a) and critical wavenumber (b) as function of $\xi$ in the case of NSBC (1) and SFBC (2).

Indeed, when a temperature fluctuation occurs in the liquid close to highly conducting wall, it easily relaxes. Whereas, it can persist and distorts the temperature distribution when the thermal conductivity of the boundary is very low. This temperature distortion can lead to an instability. As a consequence, the critical Rayleigh number is smaller [6]. When the convection flow starts, it distorts the originally horizontal isotherms. Since, this deviation from the basic state occurs in the fluid layer as well as in the wall boundary, the wavelength of the convection pattern becomes larger with decreasing $\xi$ [36]. From mathematical point of view, the decrease of $R a_{c}$ is caused by the weakening of the thermal boundary condition (29) for $\theta$ as $\xi$ decreases from $10^{3}$ to $10^{-3}$. For a given thermal conductivities ratio, the critical Rayleigh number for NSBC is greater than that for SFBC. The physical reason is quite intuitive. In the case of NSBC, the friction of the fluid against the wall dissipates more energy, therefore a hogher thermal gradient has to beimposed so that the convection can start.

Additional properties of the critical mode are given by $F_{11}$ and $G_{11}$ at the critical conditions. They are displayed in Fig. 2 for NSBC and SFBC and different values of $\xi$. As indicated above, when $\xi$ decreases, the temperature perturbation persists at the wall (Figs. 1c 1d, curves 3,2 and 1 ), reducing $R a_{c}$ and the velocity perturbation is damped (1a and $1 \mathrm{~b}$, curves 3, 2 and 1). The coupling betweeb the temperature perturbation $\theta$ in the fluid and that in the horizontal plates $\tilde{\theta}$ is illustated by Fig. 3(a), where contours of the temperature perturbation at $\xi=1$ are represented. For comparison, the case of large value of $\xi$, where the temperature vanshes in the liquid vanishes at the boundaries is also represented $(3 \mathrm{a})$. 


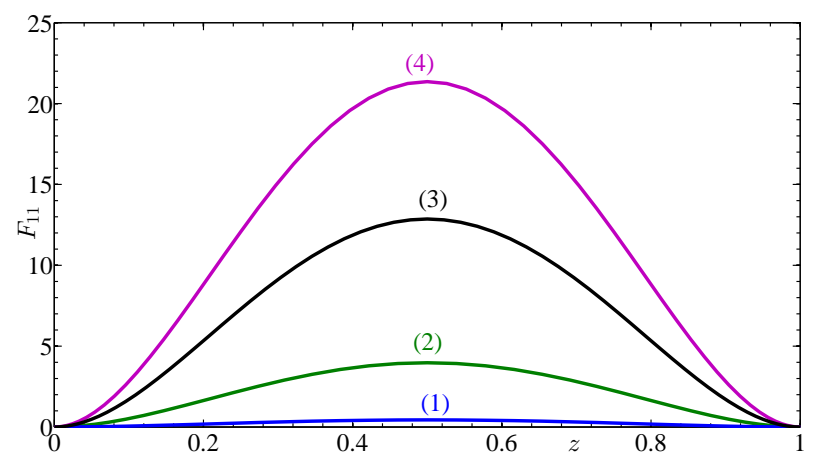

(a)

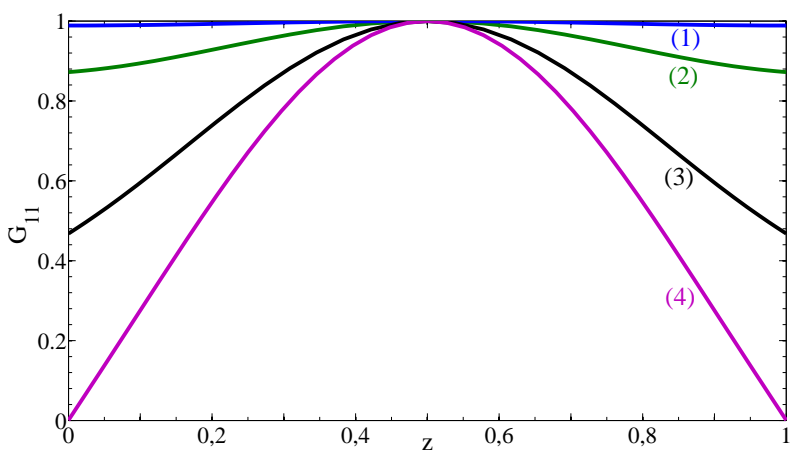

(c)

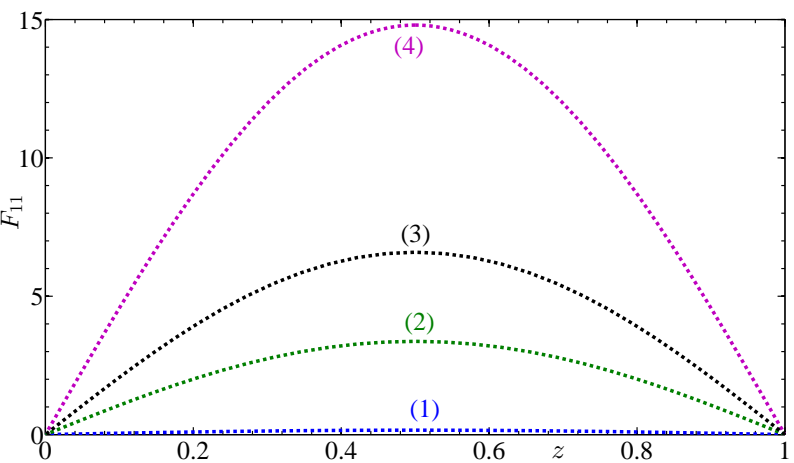

(b)

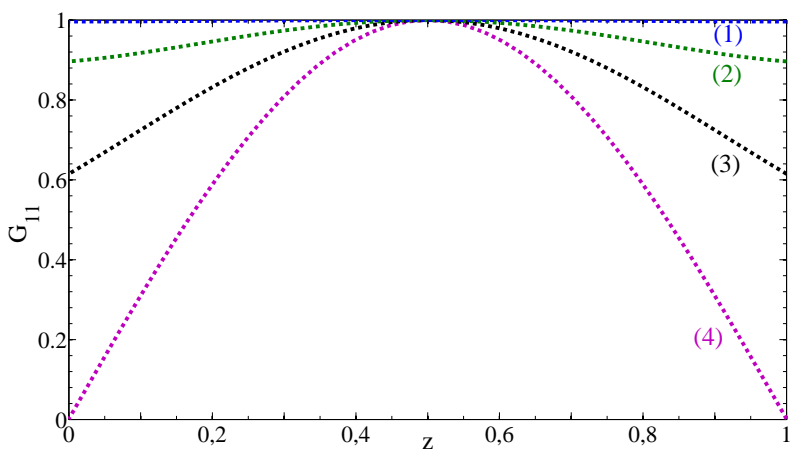

(d)

FIG. 2. Eigenfunctions at critical conditions and different values of $\xi$ : (1) $\xi=10^{-3}$; (2) $\xi=10^{-1}$; (3) $\xi=10^{0}$ and (4) $\xi=10^{3}$ in the case of NSBC (a-c) and SFBC (b-d).

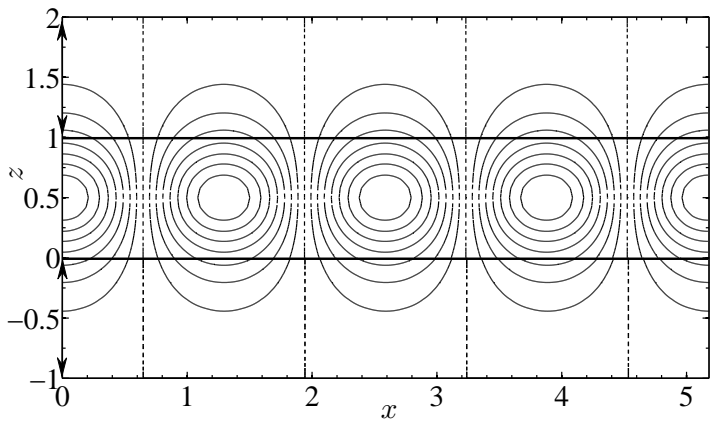

(a)

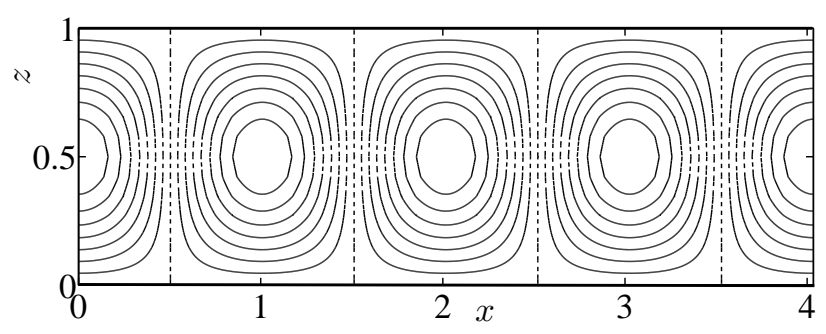

(b)

FIG. 3. Temperature perturbation contours for two values of the thermal conductivity ratio $\xi$ : (a) $\xi=1$ and (b) $\xi=1000$. In this latter case, the temperature perturbation in the liquid relaxes at the boundaries. 


\section{B. Adjoint mode}

First, we need to define an inner product between two vectors $\boldsymbol{f}$ and $\boldsymbol{g}$ by

$$
\langle\boldsymbol{f}, \boldsymbol{g}\rangle=\int_{-\Lambda}^{1+\Lambda} \boldsymbol{f} \cdot \boldsymbol{g} d z
$$

The adjoint mode $\boldsymbol{X}_{a d}$ associated to the critical mode $\boldsymbol{X}_{11}$, verifies the adjoint eigenvalue problem

$$
\boldsymbol{L}^{+} \cdot \boldsymbol{X}_{a d}=0
$$

The adjoint operator $\boldsymbol{L}^{+}$of operator $\boldsymbol{L}$ is defined by

$$
\left\langle\boldsymbol{X}_{a d}, \boldsymbol{L} \cdot \boldsymbol{X}\right\rangle=\left\langle\boldsymbol{L}^{+} \cdot \boldsymbol{X}_{a d}, \boldsymbol{X}\right\rangle
$$

\section{Characteristic time of the instability}

Near the onset of convection, the growth rate $\mathcal{R} e(s)$ of the perturbation may be approximated using Taylor expansion,

$$
s=\frac{\epsilon}{\tau_{0}}+O\left(\epsilon^{2}\right) \quad \text { with } \quad \epsilon=\frac{R a-R a_{c}}{R a_{c}} .
$$

The determination of the characteristic time follows the methodology described by Cross [9], Plaut [26]. Explicitly, it is given by

$$
\tau_{0}^{-1}=\frac{-k_{c}^{2} R a_{c}\left\langle G_{11}, F_{a d}\right\rangle}{\left\langle G_{11}, G_{a d}\right\rangle+\left\langle\tilde{G}_{11}, \tilde{G}_{a d}\right\rangle+1 / \operatorname{Pr}\left\langle\left(D^{2}-k^{2}\right) F_{11}, G_{a d}\right\rangle} .
$$

The integrals are evaluated numerically by means of Clenshaw and Curtis method, in terms of the critical conditions. Note that we consider $F_{11}=G_{11}=0$ inside the slabs and $\tilde{G}_{11}=0$ in the fluid domain. Figure 4 shows the variation of $\tau_{0}$ as a function of $\xi$. As it can be observed, The characteristic time of instability increases significantly as the thermal conductivities ratio decreases. In other words, the time needed for the system to reach a state of thermal equilibrium increases drastically with decreasing $\xi$. 


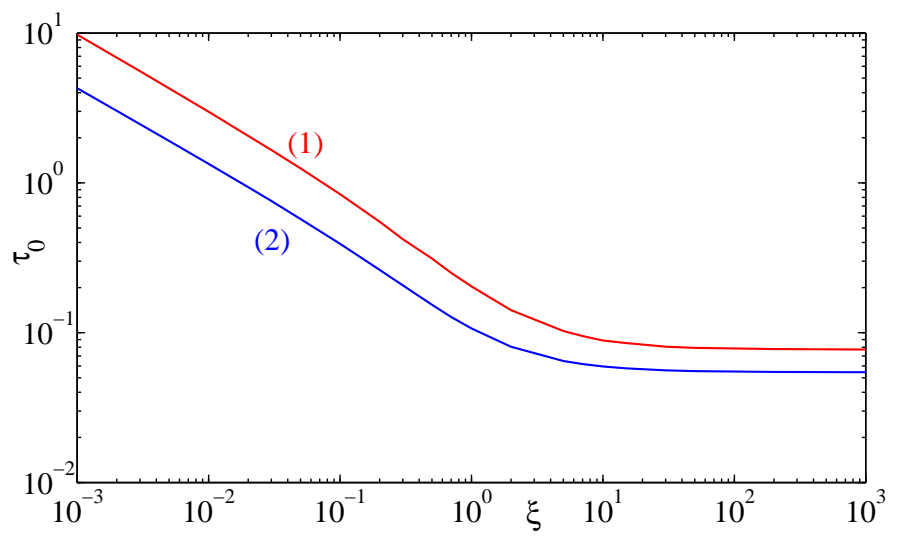

FIG. 4. Characteristic time of instability vs thermal conductivities ratio $\xi$ at $\operatorname{Pr}=10$. (1) SFBC and (2) NSBC.

\section{WEAKLY NONLINEAR STABILITY ANALYSIS}

\section{A. Principles and procedure}

A standar weakly nonlinear convection analysis using the amplitude expansion method is adopted as a first approach to investigate nonlinear effects (see Stuart [33], Watson [35], Reynolds \& Potter [29], Sen \& Venkateswarlu [31], Fujimura \& Yamada [11], Generalis \& Fujimura [12]). At leading order, one writes

$$
\begin{aligned}
w(x, y, z, t) & =f(x, y, t) F_{11}(z)+c . c ., \\
\theta(x, y, z, t) & =f(x, y, t) G_{11}(z)+c . c ., \\
\tilde{\theta}(x, y, z, t) & =f(x, y, t) \tilde{G}_{11}(z)+c . c .
\end{aligned}
$$

The planform function which describes the convection pattern is

$$
f(x, y, t)=\sum_{p=1}^{N} A_{p}(t) \exp \left(i \boldsymbol{k}_{p} \cdot \boldsymbol{r}\right)
$$

where $\left|\boldsymbol{k}_{p}\right|=k_{c}$, and $A_{p}(t)$ the amplitude of the perturbation. According to the normalization of the eigenfunctions used in the linear theory, $A_{p}(t)$ represents the amplitude of the thermal perturbation measured at the midplane. Configuration with $N=1$ corresponds to rolls and $N=2$ to squares. The weakly nonlinear analysis is applied to each of these patterns. The configuration with $N=3$ corresponding to hexagons is not considered here. Further calculations show that this three-dimensional pattern is unstable. 
In the neighborhood of the critical conditions, the dynamics are assumed to be determined by the fundamental disturbance with wavenumber $k=k_{c}$, its higher harmonics generated by the nonlinear self-interactions and the modification of the base state due to the interaction with the complex conjugate. As in Stuart [33], Watson [35], Herbert [14], the disturbance is expanded in harmonic series and the coefficient of each harmonic is further expanded in an asymptotic series with disturbance amplitude as a small parameter.

In the case of rolls, the velocity and the temperature disturbances are expanded as follows:

$$
\begin{gathered}
\left(w(x, z ; t), \theta(x, z ; t), \tilde{\theta}(x, z ; t)=\sum_{m=1}\left(F_{0,2 m}(z), G_{0,2 m}(z), \tilde{G}_{0,2 m}(z)\right)|A|^{2 m}+\right. \\
\sum_{n=1} \sum_{m=0}\left[\left(F_{n, n+2 m)}(z), G_{n, n+2 m)}(z), \tilde{G}_{n, n+2 m)}(z)\right)|A|^{2 m} A^{n} E^{n}+c . c .\right]
\end{gathered}
$$

where c.c. means the complex conjugate of its preceding expression, $E^{n}=e^{i n k_{c} x} ;, n$ denotes the harmonic index and $m$ indicates the asymptotic order. The time evolution of the amplitude $A(t)$ is given by the Stuart-Landau equation

$$
\frac{d A}{d t}=g_{0} A+g_{1}|A|^{2} A+g_{2}|A|^{4} A+\ldots
$$

In Eq. (59), $g_{0}=s$ is the linear growth rate and $g_{1}$ is the first Landau constant. The sign of $g_{1}$ determines whether the nature of the bifurcation is supercritical $\left(g_{1}<0\right)$ or subcritical $\left(g_{1}>0\right)$. Substituting expansions (58) and (59) into (21)-(23) yields after some algebra the differential equation for any $F_{n, 2 m+n}, G_{n, 2 m+n}$ and $\tilde{G}_{n, 2 m+n}$, which are solved sequentially beginning from $n=1$ and $m=0$.

The problem with harmonic index $n=1$ and amplitude order $m=0$ is the linear stability problem (35)-(37). The problem $n=0, m=1$ is $O\left(A^{2}\right)$ correction of the conductive temperature profile due to nonlinear interactions. The problem $n=2, m=0$ is the first harmonic of the fundamental mode which manifests at order $O\left(A^{2}\right)$. The problem $n=1, m=1$ is $O\left(A^{3}\right)$ correction to the fundamental mode. It contains nonhomogeneous terms due to nonlinear interations. The application of the Fredholm alternative allows the determination of the first Landau constant (the first correction to the linear growth rate) which appears in the time derivative of $w, \theta$ and $\tilde{\theta}$. It can be shown that $g_{1}$ is the sum of contributions of two terms $g_{1}^{I}$ arising from the nonlinear inertial terms and $g_{1}^{V}$ arising from the nonlinear viscous terms. Since at the lowest order $\mu=1-\alpha \Gamma$, Eqs. (13) and (14), where $\alpha=\frac{1-n_{c}}{2} \lambda^{2}$, then

$$
g_{1}=g_{1}^{I}+g_{1}^{V} \quad \text { with } \quad g_{1}^{V}-\alpha g_{1}^{N N}
$$




\section{B. Numerical method}

In the above sections, the nonlinear stability problem is reduced to a sequence of differential equations. As in the linear problem, they are solved using a spectral collocation method based on Chebyshev polynomials. The differential equations are collocated at GaussLobatto points. The integrals involved in the determination of the first Landau constant are calculated using Clenshaw and Curtis method.

\section{RESULTS AND DISCUSSION}

\section{A. Bifurcation to rolls}

The first Landau constant $g_{1}$ as well as the different contributions $g_{1}^{I}$ and $g_{1}^{N N}$ are determined for different critical sets $\left(R a_{c}, k_{c}, \xi, \operatorname{Pr}\right)$.

In Fig.5(a), we plot $g_{1}^{I}$ as a function of $\xi$. As expected, $g_{1}^{I}$ is negative, i.e., the bifurcation is supercritical for a Newtonian fluid. The absolute value of $g_{1}^{I}$ decreases with decreasing $\xi$ and $\left|g_{1}^{I}\right| \rightarrow 0$ when $\xi \rightarrow 0$. Note that $g_{1}^{I}$ is sensitive to change in $\xi$ mainly when $0.1 \leq \xi \leq 5$. For $\operatorname{Pr} \geq 1$, the analysis of the contribution to $g_{1}^{I}$ arising from the different nonlinear interactions shows that $g_{1}^{I}$ is dominated by the nonlinear thermal convection terms involving the mofication of the conductive temperature profile, for all the range of $\xi$ considered. The contribution of the nonlinear inertial term is practically negligible.

As it can be observed, $g_{1}^{N N}$ is negative, and $g_{1}^{V}=-\alpha g_{1}^{N N}>0$. Therefore, shear-thinning effects promote a subcritical bifurcation. In Fig. 6 we plot $g_{1}$ as a function of $\xi$ for different values of $\alpha$. For low shear thinning effects, $g_{1}<0$ and the bifurcation is supercritical, while for sufficiently high shear-thinning effects, $g_{1}>0$ and the bifurcation is subcritical. Using Eqs. (60), the critical degree of shear-thinning $\alpha_{c}$ above which the bifurcation changes from supercritical to subcritical is given by

$$
\alpha_{c}=\frac{g_{1}^{I}}{g_{1}^{N N}} .
$$

Figure 7 shows the variation of $\alpha_{c}$ as a function of $\xi$ at $\operatorname{Pr}=10$, for NSBC and SFBC. The more $\xi$ is low, the more the degree of shear-thinning $\alpha$ of the fluid must be high to obtain a subcritical bifurcation. This is due to the reduction of the convection intensity when $\xi$ decreases. This effect is even more pronounced in the case of SFBC. Note that, the largest 


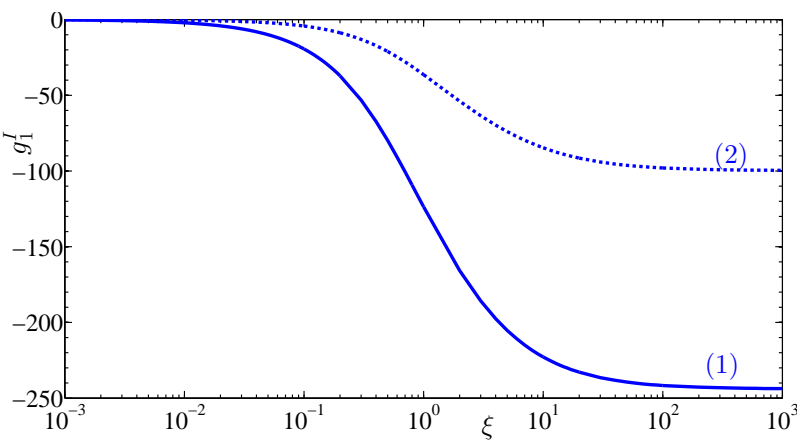

(a)

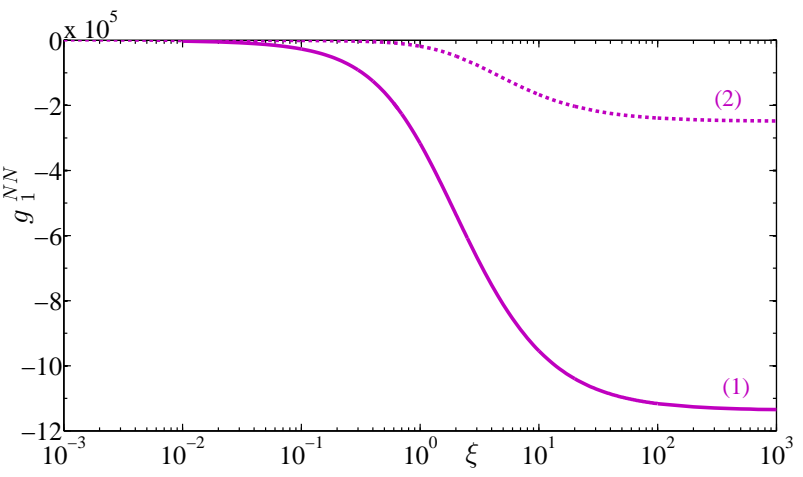

(b)

FIG. 5. Contribution of the nonlinear inertial terms (a) and nonlinear viscous terms (b) to the first cubic Landau constant, as a function of the ratio $\xi$ at $\operatorname{Pr}=10$. Curves (1) and (2) correspond to NSBC and SFBC respectively.

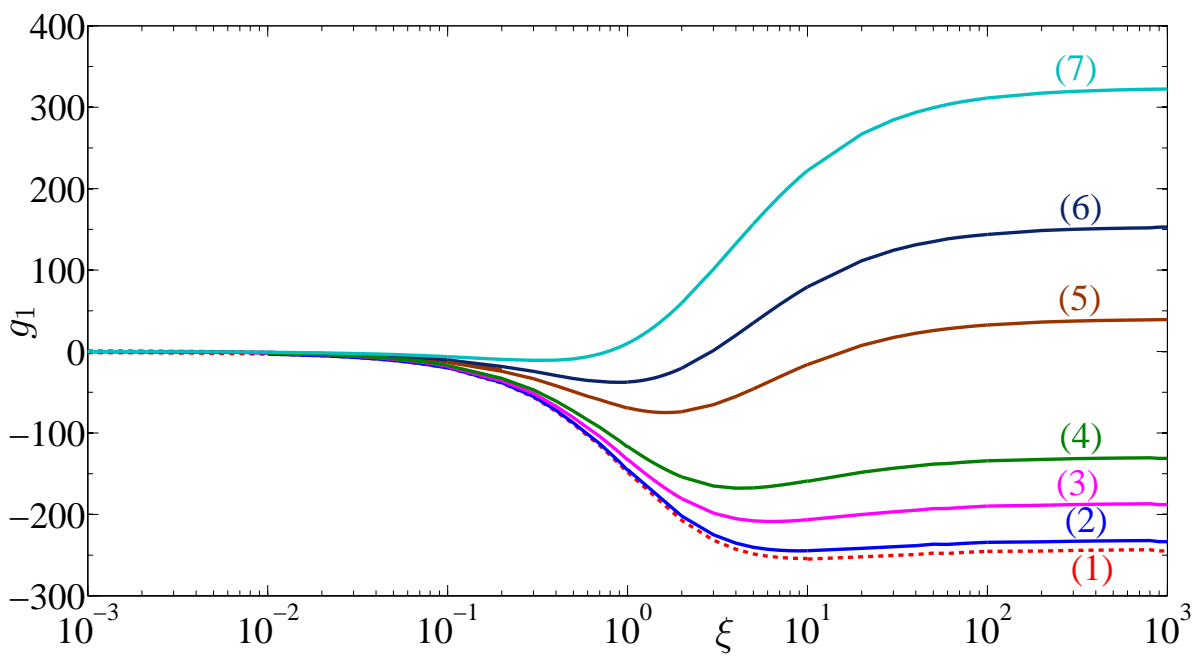

FIG. 6. Cubic Landau constant as a function of $\xi$ in the case of NSBC with $\operatorname{Pr}=10$ and different values of $\alpha$ : (1) $\alpha=0$, i.e. Newtonian case ; (2) $\alpha=1 \times 10^{-5}$; (3) $\alpha=5 \times 10^{-5}$; (4) $\alpha=10^{-4}$; (5) $\alpha=2.5 \times 10^{-4} ;(6) \alpha=3.5 \times 10^{-4} ;(7) \alpha=5 \times 10^{-4}$.

variation of $\alpha_{c}$ with $\xi$ occurs mainly for $10^{-2} \leq \xi \leq 10$. 

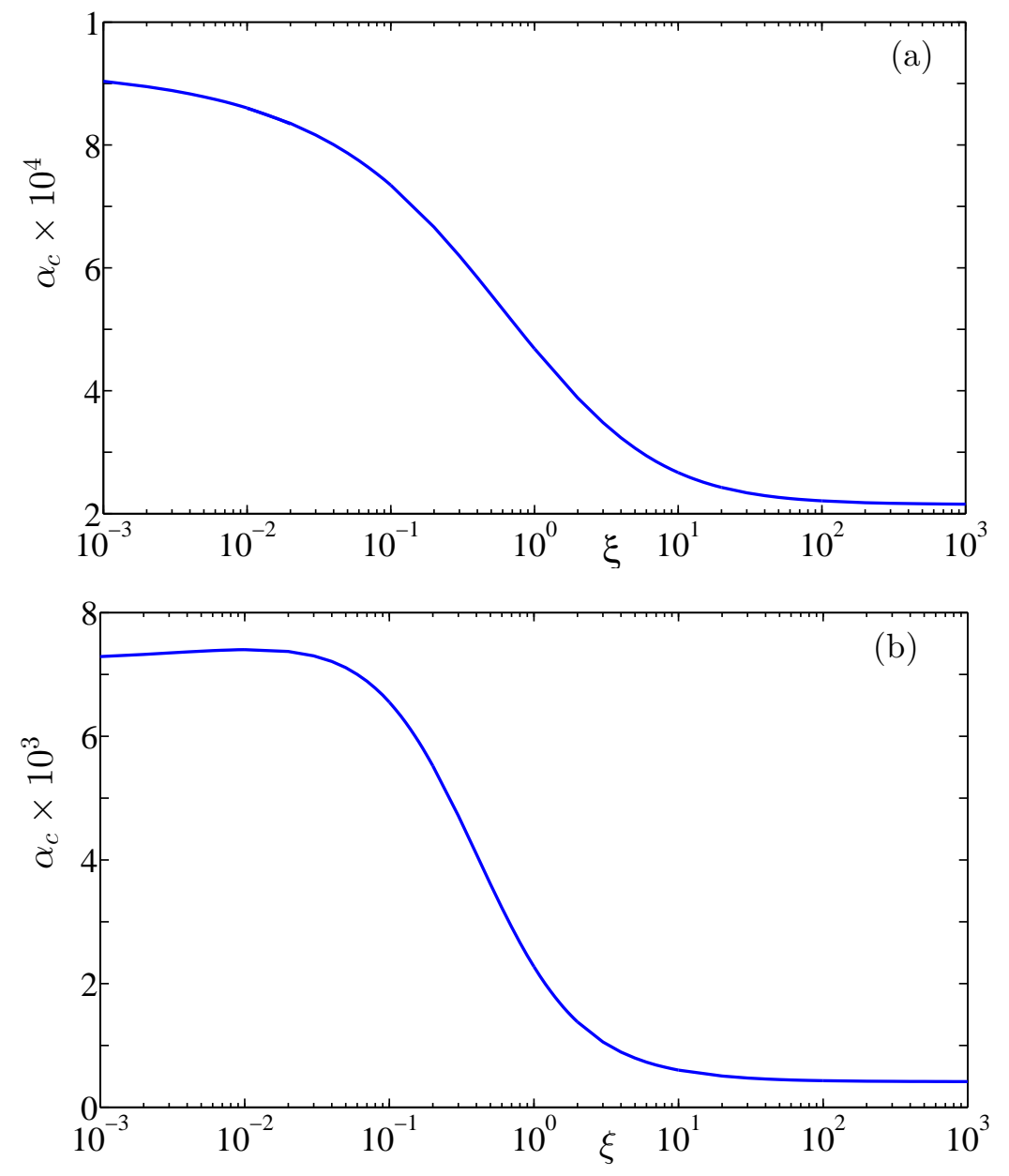

FIG. 7. For rolls, critical value of the degree of shear-thinning $\alpha_{c}$, vs the thermal-conductivities ratio $\xi$ at $\operatorname{Pr}=10$ for (a) NSBC and (b) SFBC.

\section{Bifurcation to squares}

For square patterns, considered as the superposiution of two perpensicular sets of rolls, the amplitude equations are [13]:

$$
\begin{aligned}
& \frac{d A_{1}}{d t}=s A_{1}+\left[g_{1} A_{1}^{2}+\lambda_{1} A_{2}^{2}\right] A_{1}, \\
& \frac{d A_{2}}{d t}=s A_{2}+\left[g_{1} A_{2}^{2}+\lambda_{1} A_{1}^{2}\right] A_{2} .
\end{aligned}
$$

As for $g_{1}$, the coefficient of cross saturation $\lambda_{1}$ is obtained by invoking the solvability condition and can be written as:

$$
\lambda_{1}=\lambda_{1}^{I}-\alpha \lambda_{1}^{N N}
$$



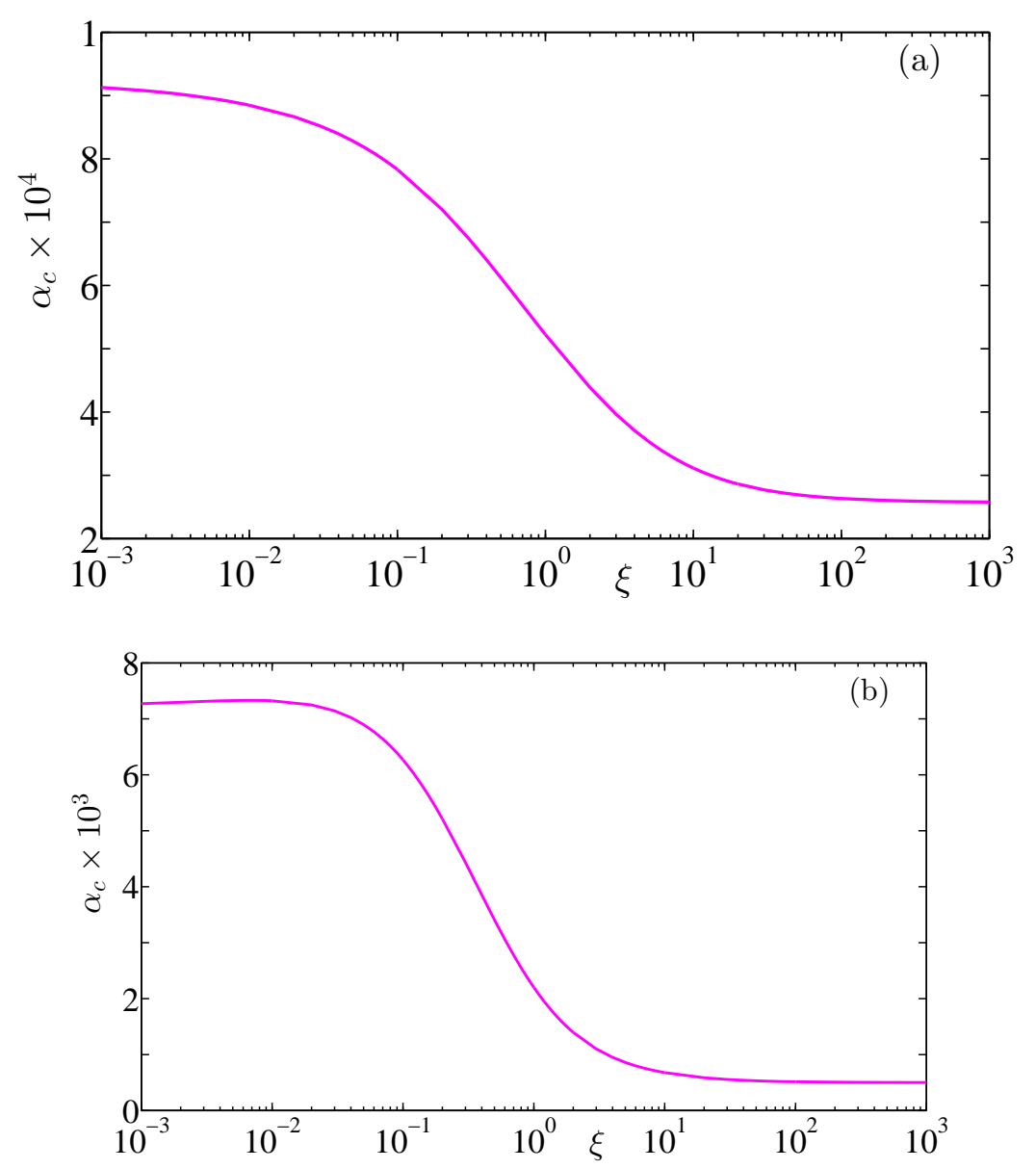

FIG. 8. For squares, critical value of the degree of shear-thinning $\alpha_{c}$, vs the thermal-conductivities ratio $\xi$ at $\operatorname{Pr}=10$ for (a) NSBC and (b) SFBC.

The numerical results indicate that $\lambda_{1}^{I}$ and $\lambda_{1}^{N N}$ are negative and their variation with $\xi$ is similar to that of $g_{1}^{I}$ and $g_{1}^{N N}$ respectively. The critical value of $\alpha$ above which, the bifurcation becomes subcritical is given by

$$
\alpha_{c}=\frac{g_{1}^{I}+\lambda_{1}^{I}}{\left(g_{1}^{N N}+\lambda_{1}^{N N}\right)}
$$

The variation of $\alpha_{c}$ as a function of $\xi$, at $\operatorname{Pr}=10$, is depicted in Fig. 8 for NSBC and SFBC. As in the case of rolls, $\alpha_{c}$ increases with decreasing $\xi$.

The influence of Prandtl number on $\alpha_{c}$ is shown in Figure 9 for two limit values of $\xi$ : $10^{-3}$ and $10^{3}$. Note that for $\operatorname{Pr} \geq 10, \alpha_{c}$ no longer depends on $\operatorname{Pr}$. However, when $\operatorname{Pr}<1$, $\alpha_{c}$ increases strongly as $\operatorname{Pr}$ decreass. 


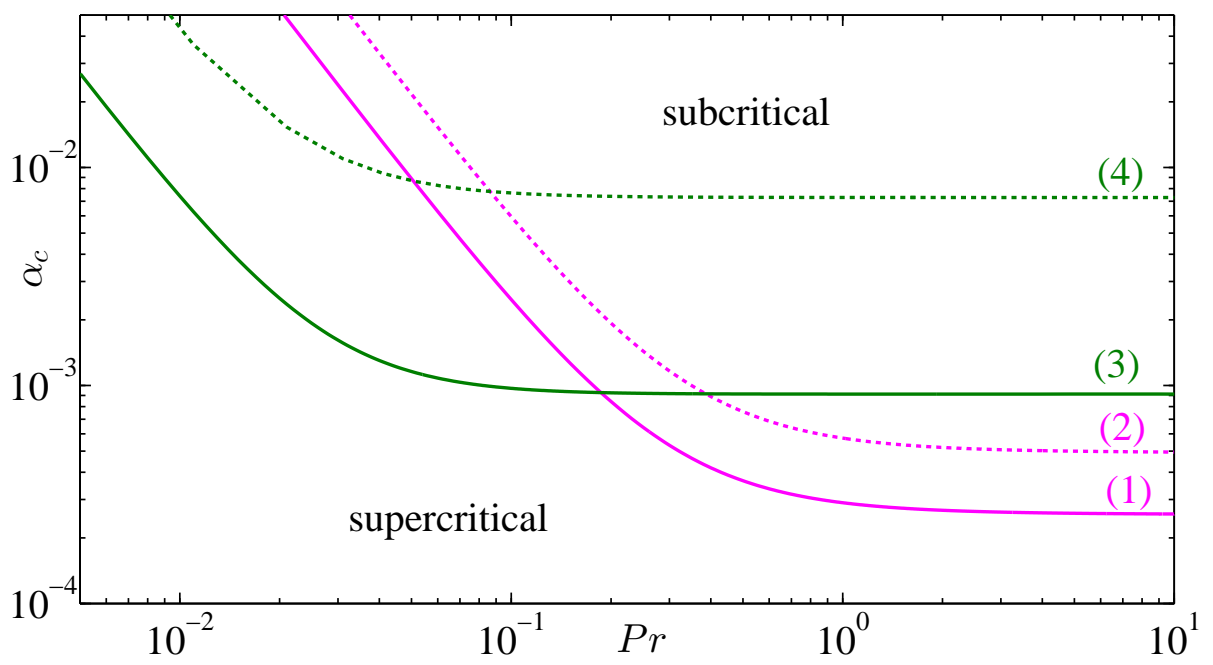

FIG. 9. Critical value of the degree of shear-thinning $\alpha_{c}=\left(\left|\frac{d \mu}{d \Gamma}\right|_{\Gamma=0}\right)_{c}$ as a function of Prandtl number. Case of square patterns. (1) NSBC with $\xi=10^{3} ;(2)$ SFBC with $\xi=10^{3} ;$ (3) NSBC with $\xi=10^{-3} ;$ (4) SFBC with $\xi=10^{-3}$.

\section{B. Pattern selection}

In this section, we investigate the pattern selection on a square lattice. The calculation proceeds in two stages. First, the possible steady states solutions of the amplitude equations are determined. Then, their linearized stability is determined by computing the eigenvalues of the linearized system around each solution. A solution is stable if all its eigenvalues are negative.

The system of amplitude equations for a square lattice are given by Eqs (62) and (63). The coefficients $s, g_{1}$ and $\lambda_{1}$ in these equations depend on the rheological parameters and on the reduced Rayleigh number $\epsilon=\left(R a-R a_{c}\right) / R a_{c}$. The stationary solutions are obtained by setting $f_{i}\left(A_{1}, A_{2}\right)=0$, where $f_{i}$ is the right hand side of the amplitude equations. Their stability is determined by the sign of the eigenvalues $\chi_{i}$ of the Jacobian matrix $\boldsymbol{J}_{i j}=\frac{\partial f_{i}}{\partial A_{j}}$ evaluated at the steady states. In the following, the stability of the stationary solutions is examined in details.

(i) Conduction state, $A_{1}=A_{2}=0$. The eigenvalues associated to this state are $\chi_{1}=\chi_{2}=s$. The conduction state is stable if $\epsilon<0$ and undergoes a stationary bifurcation at $\epsilon=0$.

(ii) Steady convection with rolls parallel to $\boldsymbol{e}_{x}$ or $\boldsymbol{e}_{y}, A_{1}=\sqrt{-s / g_{1}}, A_{2}=0$ or $A_{1}=0, A_{2}=$ 


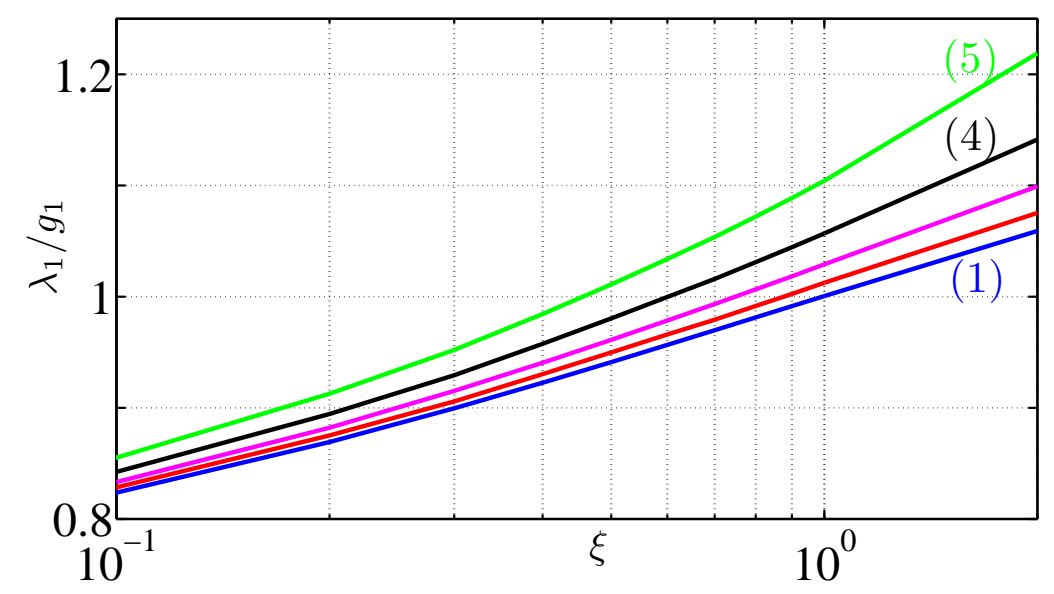

FIG. 10. Ratio of the cross saturation coefficient $\lambda_{1}$ to the saturation coefficient $g_{1}$ versus the ratio of the thermal conductivities $\xi$ for different values of the shear-thinning degree $\alpha$ in the case of NSBC. (1) $\alpha=0 ;(2) \alpha=2.5 \times 10^{-5}$; (3) $\alpha=5.625 \times 10^{-5}$; (4) $\alpha=10^{-4}$; (5) $\alpha=1.5625 \times 10^{-4}$.

$\sqrt{-s / g_{1}}$. The eigenvalues associated to this state are: $\chi_{1}=-2 s$ and $\chi_{2}=s \frac{g_{1}-\lambda_{1}}{g_{1}}$.

(iii) Steady convection with square patterns, $A_{1}=A_{2}=\sqrt{-s /\left(g_{1}+\lambda_{1}\right)}$. The eigenvalues associated to this steady state are $\chi_{1}=-2 s$ and $\chi_{2}=\frac{2 s\left(\lambda_{1}-g_{1}\right)}{\lambda_{1}+g_{1}}$.

In the supercritical regime, i.e. $s>0$ and $\chi_{1}<0$. The sign of $\chi_{2}$ depends on the ratio $\lambda_{1} / g_{1}$. It is represented in Fig. 10 as a function of $\xi$ for different values of $\alpha$. When $\left|\lambda_{1}\right|>\left|g_{1}\right|$, $\chi_{2}$ is positive and the squares are unstable. According to [10], the interaction between the two sets of rolls is too strong and one of the two sets of rolls nonlinearly damps out the other. When $\left|\lambda_{1}\right|<\left|g_{1}\right|, \chi_{2}<0$ and the squares are stable. The critical value of $\xi$ at which the planform of convection changes from square-cell solution $\left(\xi<\xi_{c}\right)$ to two-dimensional roll solution $\left(\xi>\xi_{c}\right)$ is given as a function of $\alpha$ in Fig. 11. In the Newtonian case, i.e. $\alpha=0, \xi_{c}=1$. This result is in agreement with that given by Jenkins and Proctor [17]. With increasing shear thinning effects, the interaction between the two sets of rolls of a square-cell increases, reducing by this way, $\xi_{c}$.

\section{SOLUTIONS AT HIGHER ORDER}

Figure 11 is obtained by truncating the series (59) to the first Landau constant, i.e. at cubic order in $A$. For a significant deviation from the critical conditions, terms of higher 


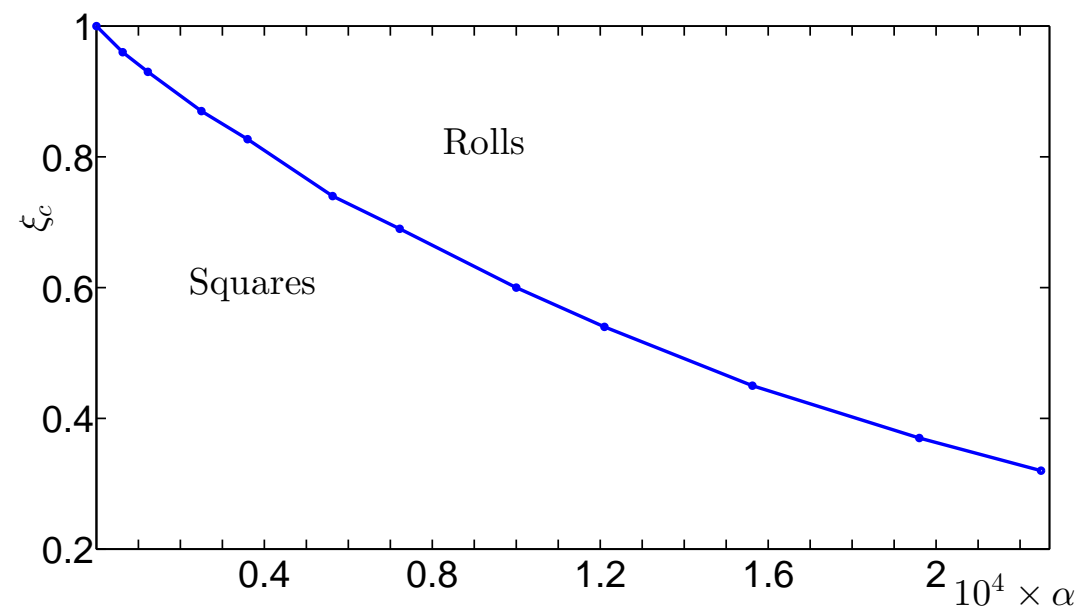

FIG. 11. $\xi_{c}$ as a function of $\alpha$ for NSBC with $\operatorname{Pr}=10$ and $\Lambda=1$. The planform of convection is a square-cell, when $\xi<\xi_{c}$, and a two-dimensional roll when $\xi>\xi_{c}$

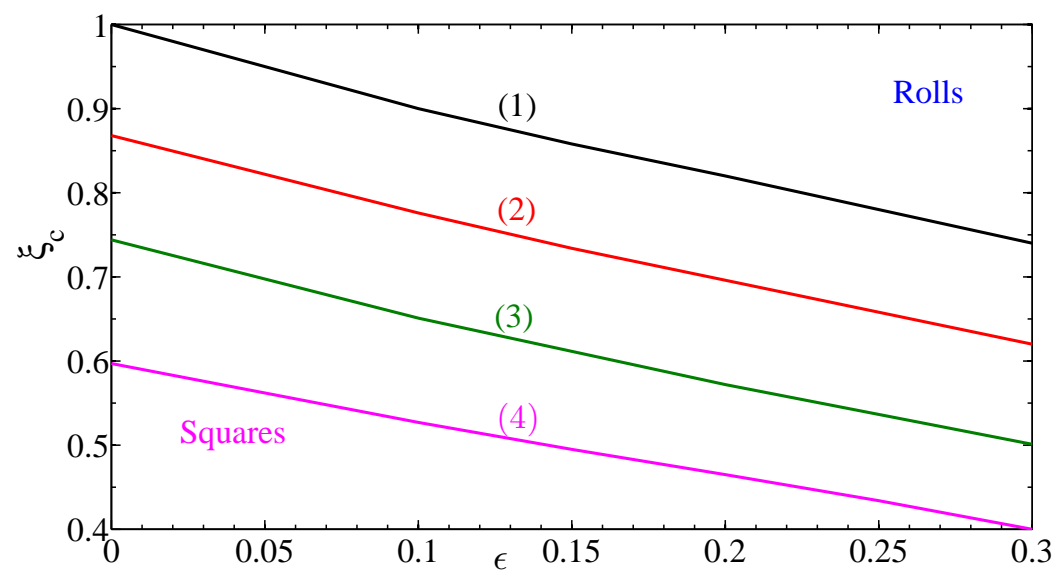

FIG. 12. $\xi_{c}$ as a function of $\epsilon$ for NSBC with $n_{c}=0.5, \operatorname{Pr}=10$ and $\Lambda=1$. Squares are stable for $\xi<\xi_{c}$, and rolls are stable for $\xi>\xi_{c} .(1): \lambda=0,(2): \lambda=0.01,(3): \lambda=0.015,(4): \lambda=0.02$.

order become large and should be taken into account. A weakly nonlinear expansion was then carried out up to fifth-order in amplitude. Figure 12 shows the evolution of $\xi_{c}$ versus the reduced Rayleigh number, $\epsilon$, for different values of the constant time of the fluid $\lambda$. The shear-thinning index is fixed to $n=0.5$. The intensity of convection increases with increasing $\epsilon$. The interaction between the two sets of rolls, via nonlinear inertial and nonlinear viscous terms become stronger leading to a diminution of $\xi_{c}$. 


\section{HEAT TRANSFER, FLOW STRUCTURE AND VISCOSITY FIELD}

In the present section, information on the heat transfer, the flow structure and viscosity field in rolls and squares are provided. The influence of shear-thinning will be emphasized.

\section{A. Heat transfer}

The heat transfer through the horizontal fluid layer is described by the Nusselt number, $N u$, the ratio of the total heat flux to the purely conductive heat flux in the absence of fluid flow. It can be calculated either at the lower or upper plate. At the lower plate, we have

$$
N u=1-\left(\frac{\partial \bar{\theta}}{\partial z}\right)_{z=0}=1-\sum_{p=1}^{N} \sum_{m=1}^{M}\left[A_{p}^{2 m}\left(D G_{02 m}\right)_{z=0}\right]-S A_{1}^{2} A_{2}^{2}\left(D \underline{G}_{04}\right)_{z=0}
$$

where the overbar denotes the horizontal average over one wavelength, $N=1$ corresponds to rolls and $N=2$ to squares, $M=1$, when the series (59) is truncated at the third order, and $M=2$, when (59) is truncated at the fifth order, $S=1$ for squares and $S=0$ for rolls. The term $D \underline{G}_{04}$ arises from the interaction between modes with different eigenvectors. The unperturbed solution, $N u=1$, corresponds to the hydrostatic solution. The second term of $N u$ refers to the convective transfer.

Figure 13 shows for a two-dimensional roll solution computed at the fifth-order, the evolution of $N u-1$ as a function of $\epsilon$ for different values of $\xi$. The Nusselt number decreases with decreasing $\xi$. When $\xi \rightarrow 0$, i.e. for poorly conducting plates, $N u \rightarrow 1$. The convection in the fluid layer will not contribute to the overall heat transfer. For $\xi=1000$, to represent the case of perfectly conducting walls, our results are in good agreement with the numerical solution of (21) and (22) obtained using the spectral code of [27], at least up to $\epsilon=0.25$. The influence of shear-thinning effects is illustrated by the dotted curves. The Nusselt number increases with increasing shear-thinning effects (Pierre \& Tien [25], Liang \& Acrivos [22], Ozoe \& Churchill [24], Lamsaadi et al. [20], Aloui et al. [1], Bouteraa et al. [3]. For low values of $\xi$, the influence of shear-thinning effects is reduced. Figure 14 shows $N u-1$ as a function of $\epsilon$ for rolls and squares at two values of $\xi: 0.1$ and 1. At $\xi=0.1$, the Nusselt number is larger for squares than for rolls, while at $\xi=1, N u$ is greater for rolls than for squares. The differences are small but notable and in agreement with the maximum heat transfert principle: the only stable solution is the one of maximum heat transport (Malkus 


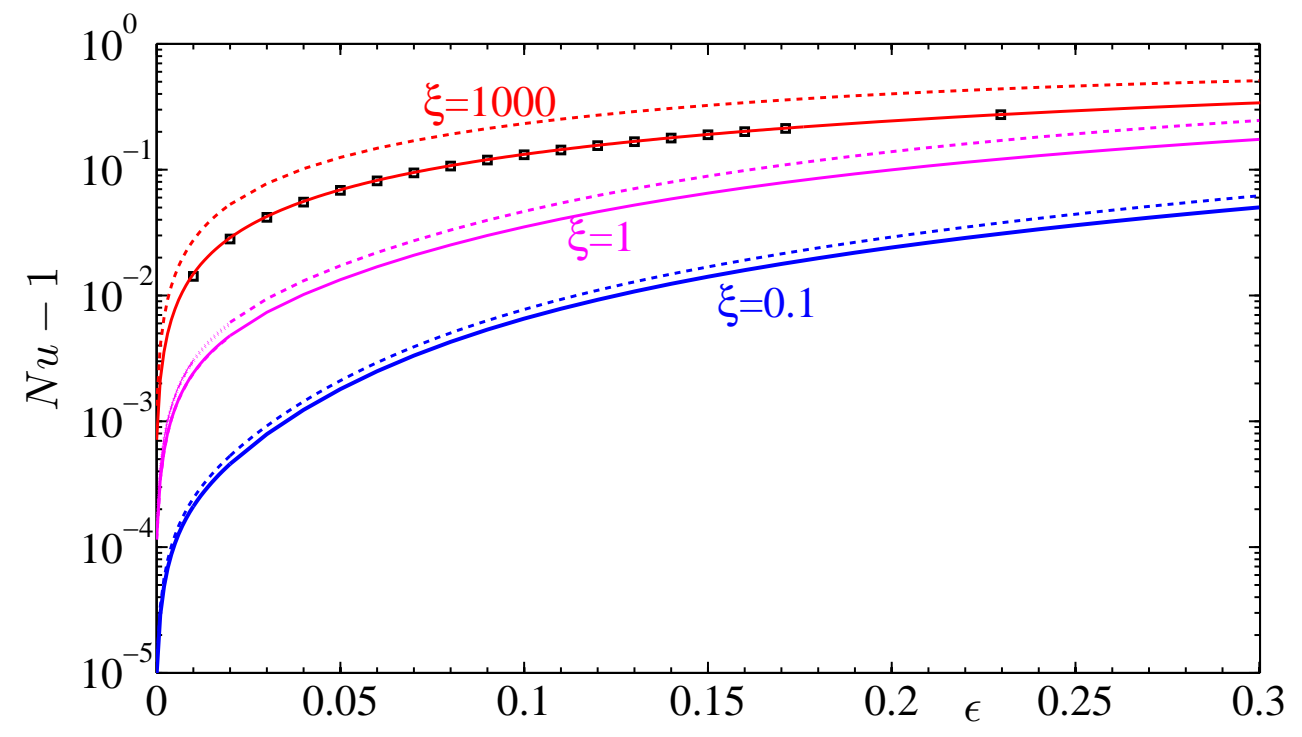

FIG. 13. Nusselt number as a function of the reduced Rayleigh number $\epsilon$ in the case of NSBC at $\operatorname{Pr}=10$ and for three values of $\xi$. The Continuous lines correspond to a Newtonian fluid and the dashed lines to a shear-thinning fluid with $n=0.5, \lambda=0.02$; ( $\square$ ) numerical solution of (21) and (22) obtained using the spectral code of Plaut \& Busse [27] in the case of a Newtonian fluid with perfectly conducting walls.

\& Veronis [23])

\section{B. Viscosity field}

The viscosity distribution for rolls and square cells, computed at the fifth order in amplitude, is shown in Fig. 15 for $\epsilon=0.2$. Two values of $\xi$ are considered. The first one is $\xi=1000$, where rolls are stable and the second one is $\xi=0.1$ where squares are stable. For the rheological parameters, we set $n$ and $\lambda$ such that $\alpha=0.2 \alpha_{c}$.

Rolls, $\xi=1000$ with NSBC. The viscosity field and the flow structure are illustrated by Fig 15 (a). The interior of the roll is practically isoviscous with $\mu \approx 1$. The viscosity is minimal at the wall where the shear-rate is maximal. It is weakly reduced at the four corners of the roll because of the elongational rate $\dot{\gamma}_{z z}=-\dot{\gamma}_{x x}$.

Squares, $\xi=0.1$ with NSBC. Because of the symmetry of the solution, considered as a superposition of two equal strength perpendicular roll sets in the $x$ - and $y$-directions, no 

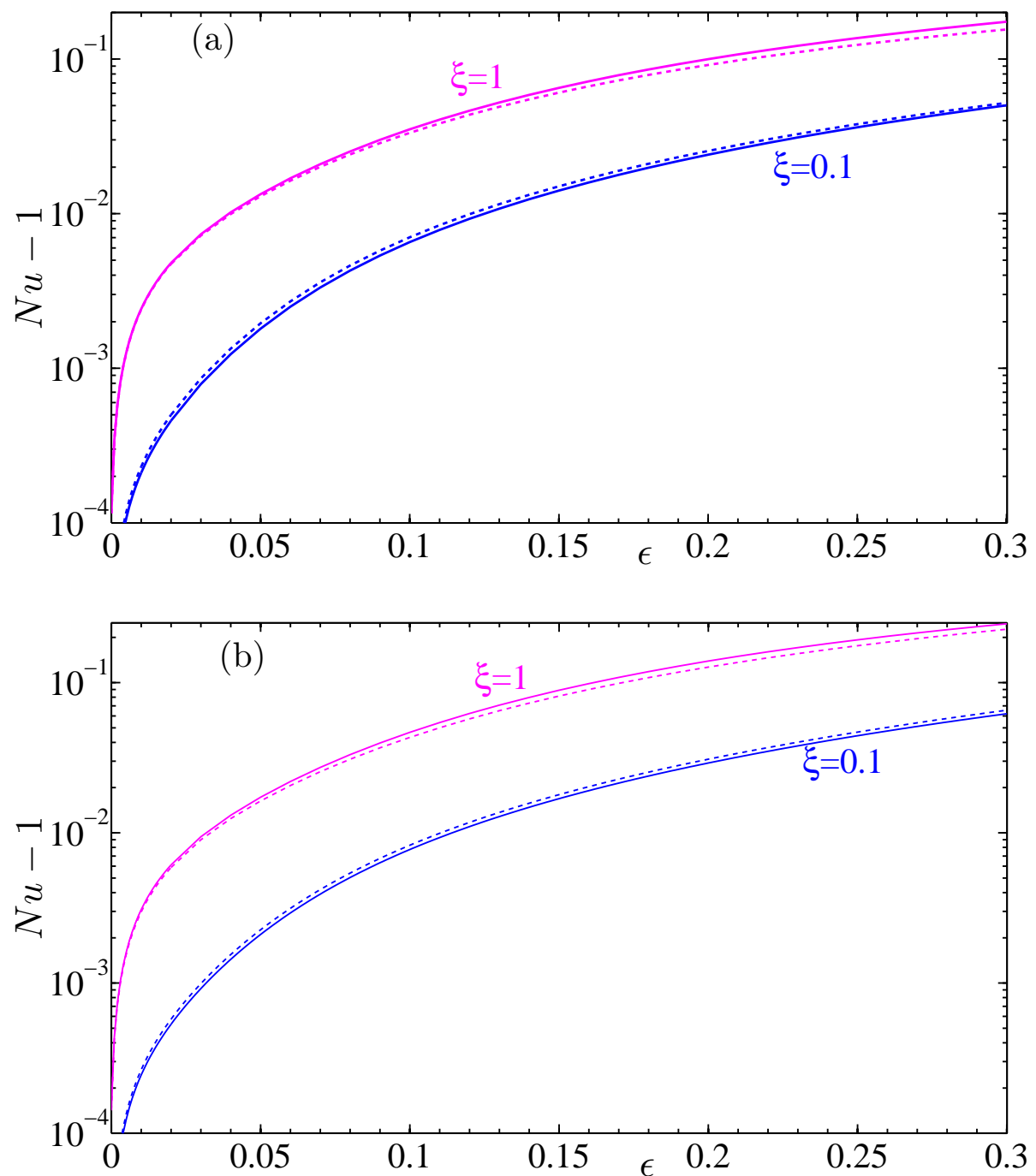

FIG. 14. Nusselt number as a function of the reduced Rayleigh number $\epsilon$ in the case of NSBC with $\operatorname{Pr}=10$ and for two values of $\xi$. (a) Newtonian fluid, (b) shear-thinning fluid with $n=0.5$ and $\lambda=0.02$. The Continuous lines correspond to rolls and the dashed lines to squares.

fluid passes through the vertical diagonal planes and the vertical cell boundaries. Since the amplitude equations on a square lattice involve $A$ in the form of $A^{2}$, there are two equiprobable opposite motions (as for rolls). In the first one, the fluid is downwardly directed in the cell centre and upwardly through the vertical cell boundaries. In the second one, the fluid is upwardly directed in the cell centre and downwardly through the vertical cell boundaries. We have chosen to represent the second case. The vertical velocity profile is shown in Fig. 15(c) for NSBC ans SFBC. The viscosity field and the flow structure are 
(a)

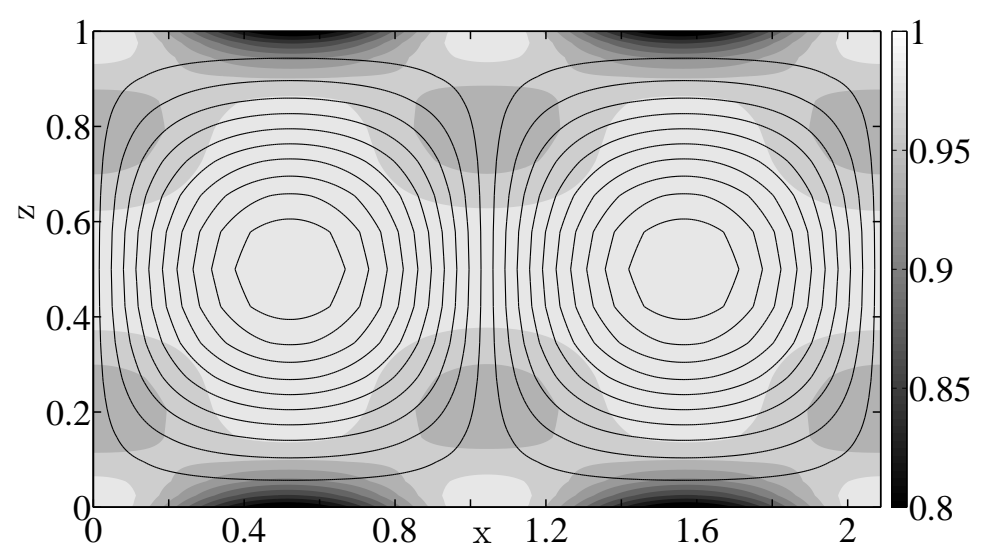

(b)

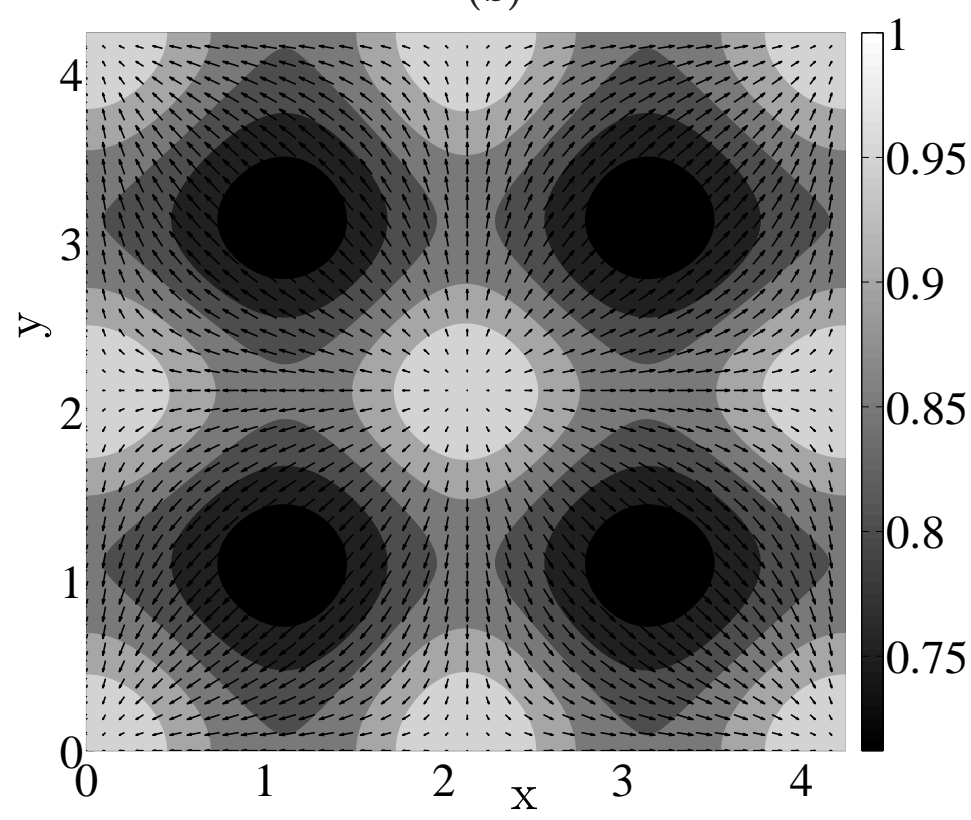

(c)

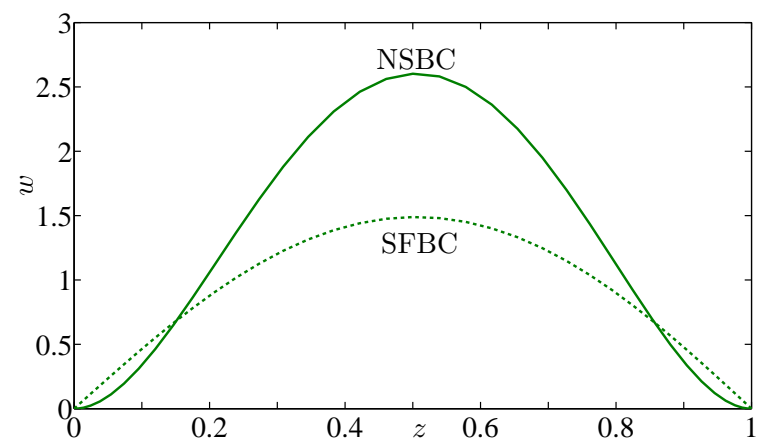

FIG. 15. Flow structure and viscosity distribution over a roll $(\mathbf{a}, \xi=1000)$ and a square (b, $\xi=0.1)$ at $\epsilon=0.2$, with NSBC, $\alpha=0.2 \alpha_{c}$ and $\operatorname{Pr}=10$. For the square, the horizontal velocity field is shown at $z=0.8$. (c) Vertical velocity profile in the square cell centre for NSBC and SFBC. 
displayed in Fig. 15(b). At the wall and around the stagnation points (light region in the figure), the viscosity is weakly reduced. The viscosity is minimal at locations (dark region in the figure) where the shear rates $\dot{\gamma}_{x z}$ and $\dot{\gamma}_{y z}$ are maximal.

\section{CONCLUSION}

We have investigated the influence of shear-thinning effects on the convection in a horizontal layer of a shear-thinning fluid between two horizontal symmetric plates of finite thermal conductivity. The rheological behavior of the fluid is described by the Carreau model. The critical Rayleigh number $R a_{c}$ and wavenumber $k_{c}$ for the onset of convection are determined as a function of the ratio $\xi$ of the thermal conductivity of the plates to that of the fluid. As the fluid viscosity at zero shear rate is constant, the values of $R a_{c}$ and $k_{c}$ in NSBC and SFBC are in very good quantitative agreement with those given in the literature for a Newtonian fluid. Additional results dealing with the characteristic time of instability $\tau_{0}$ are provided. It is found that $\tau_{0}$ increases significantly when $\xi<1$. The nature of the bifurcation to rolls and squares has been determined using a three-dimensional weakly nonlinear approach of amplitude equations. The critical value of the shear-thinning degree $\alpha_{c}$ above which the bifurcation becomes subcritical is determined as a function of $\xi$. It is shown that $\alpha_{c}$ increases with decreasing $\xi$. This variation occurs mainly for $10^{-2} \leq \xi \leq 10$. For $\xi>10, \alpha_{c}$ is practically the same as for perfectly conducting plates. The numerical results indicate also that $\alpha_{c}$ is independent of $\operatorname{Pr}$ when $\operatorname{Pr}>10$. The stability of rolls and squares is then investigated as a function of $\xi$ and the rheological parameters. In the Newtonian case, squares are stable when $\xi<1$, in agreement with [17]. In the case of shear-thinning fluids, an additional nonlinear coupling between modes is introduced by the rheological law. This leads to a decrease of the crtical value of $\xi$ below which squares are stable.

By considering the amplitude expansion at the fifth order, the range of validity of the weakly nonlinear analysis is extended and the domain of stability of the square pattern in the $(\xi, \epsilon)$ plane is determined. These results are consistent with the maximum heat-transfer principle.

In the weakly nonlinear approach, it is assumed implicitly that the dynamics is dominated by the fundamental mode. We intend to analyse the stability of the convective patterns, in 
the space Rayleigh-wavenumber, as a function of $\xi$ and shear-thinning effects.

\section{Acknowledgements}

The authors gratefully acknowledge the financial support of the French National Research Agency, under the grant ANR-10-BLAN-925-01. 
[1] Z. Alloui, N. Ben Khelifa, H. Beji, P. Vasseur, and A. Guizani. The onset of convection of power-law fluids in a shallow cavity heated from below by a constant heat flux. J. NonNewtonian Fluid. Mech., 196:70-82, 2013.

[2] R. B. Bird, R. Amstrong, and O. Hassager. Dynamics of polymeric liquids. Wiley-Interscience, New York, 1987.

[3] M. Bouteraa, C. Nouar, E. Plaut, C. Métivier, and A. Kalck. Weakly nonlinear analysis of Rayleigh-Bénard convection in shear-thinning fluids: nature of the bifurcation and pattern selection. J. Fluid. Mech, 767:696-734, 2015.

[4] F. H. Busse and N. Riahi. Nonlinear convection in a layer with nearly insulating boundaries. J. Fluid. Mech, 96(02):243-256, 1980.

[5] P. Carriere, A. Bottaro, and P. Metzener. Wavelength selection in Rayleigh-Bénard convection between horizontal boundaries of finite conductivity. Eur. J. Mech. B/Fluids, 16:483-508, 1997.

[6] P. Cerisier, S. Rahal, J. Cordonnier, and G. Lebon. Thermal influence of boundaries on the onset of Rayleigh-Bénard convection. Int. J. Heat. Mass Transfer, 41:3309-3320, 1998.

[7] C. J. Chapman and M. R. E. Proctor. Nonlinear Rayleigh-Bénard convection between poorly conducting boundaries. J. Fluid. Mech, 101(04):759-782, 1980.

[8] R. M. Clever and F. H. Busse. Convection in a layer heated from below with a nearly insulating boundary. Phys. Rev. E, 57:4198-4205, 1998.

[9] M.C. Cross. Derivation of the amplitude equation at the Rayleigh-Bénard instability. Phys. Fluids., 23(9):1727-1731, 1980.

[10] S. Fauve. Pattern forming instabilities. Cambridge University Press, In Hydrodynamics and nonlinear instabilities, edited by P. Huerre and M. Rossi, 1998.

[11] K. Fujimura and S. Yamada. Hexagons and triangles in the Rayleigh-Bénard problem: quinticorder equations on a hexagonal lattice. Proc. R. Soc. A, 464:2721-2739, 2008.

[12] S.C. Generalis and K. Fujimura. Range of validity of weakly nonlinear theory in the RayleighBénard problem. J. Phys. Soc. Jpn, 78:1-11, 2009.

[13] M. Golubitsky, J.W. Swift, and E. Knoblock. Symmetries and pattern selection in RayleighBénard convection. Physica D., 10:249-276, 1984. 
[14] T. Herbert. On perturbation methods in nonlinear stability theory. J. Fluid. Mech, 126:167$186,1983$.

[15] B. Holmedal, M. Tveitereid, and E. Palm. Planform selection in Rayleigh-Bénard convection between finite slabs. J. Fluid. Mech, 537:255-270, 2005.

[16] D. T. J. Hurle, E. Jakeman, and E. R. Pike. On the solution of the Bénard problem with boundaries of finite conductivity. Proc. R. Soc. Lond A, 296:469-475, 1967.

[17] D. R. Jenkins and M. R. E. Proctor. The transition from roll to square-cell solutions in Rayleigh-Bénard convection. J. Fluid. Mech, 139:461-471, 1984.

[18] Z. Kebiche. Etude expérimentale de l'instabilité de Rayleigh-Bénard dans les fluides nonNewtoniens. Ph.D. thesis, Université de Nantes, 2014.

[19] Z. Kebiche, C. Castelain, and T. Burghelea. Experimental investigation of the RayleighBénard convection in a yield stress fluids. J. Non-Newtonian Fluid Mech., 203:9-23, 2014.

[20] M. Lamsaadi, M. Naimi, and M. Hasnaoui. Natural convection of power law fluids in a shallow horizontal rectangular cavity uniformly heated from below. Heat Mass Transfer, 41:239-249, 2005 .

[21] P. LeGal and V. Croquette. Appearance of a square pattern in a Rayleigh-Bénard experiment. Phys. Fluids, 31(11):3440-3442, 1988.

[22] S. F. Liang and A. Acrivos. Experiments on buoyancy-driven convection in non-newtonian fluids. Rheol. Acta., 9:447-455, 1970.

[23] W. V. R. Malkus and G. Veronis. Finite amplitude cellular convection. J. Fluid. Mech, 4:225-260, 1958.

[24] H. Ozoe and S. W. Churchill. Hydrodynamic stability and natural convection in newtonian and non-newtonian fluids heated from below. AIChE Symposium Series, 69:126-133, 1973.

[25] St. C. Pierre and C. Tien. Experimental investigation of natural convection heat transfer in confined space for non-newtonian fluid. Can. J. Chem. Eng, 41:122-127, 1963.

[26] E. Plaut. Modélisation d'instabilités par méthodes nonlinéaires. Cours Master 2, Université de Lorraine, 2008.

[27] E. Plaut and F. H. Busse. Low-Prandtl-number convection in a rotating cylindrical annulus. J. Fluid. Mech, 464:345-363, 2002.

[28] R.E. Proctor. Planform selection by finite amplitude thermal convection between poorly conducting slabs. J. Fluid. Mech, 113:469-485, 1981. 
[29] W. C. Reynolds and M. C. Potter. Finite amplitude instabillity of parrallel shear flows. J. Fluid. Mech, 27:465-492, 1967.

[30] N. Riahi. Nonlinear convection in a porous layer with finite conducting boundaries. J. Fluid. Mech, 129:153-171, 1983.

[31] P.K. Sen and D. Venkateswarlu. On the stability of plane Poiseuille flow to finite-amplitude disturbances, considering the higher-order Landau coefficients. J. Fluid. Mech, 133:179-206, 1983.

[32] E.M. Sparrow, R.J. Goldstein, and V.K. Jonsson. Thermal instability in a horizontal fluid layer: effect of boundary conditions and a non-linear temperature profile. J. Fluid. Mech, 18(04):513-528, 1964.

[33] J. T. Stuart. On the non-linear mechanics of wave disturbances in stable and unstable parallel flows. part 1. the basic behaviour in plane Poiseuille flow. J. Fluid. Mech, 9:353-370, 1960.

[34] R. Tanner. Engineering rheology. Oxford University Press, New York, 2000.

[35] J. Watson. On the non-linear mechanics of wave disturbances in stable and unstable parallel flows. part 1. the development of a solution for plane Poiseuille flow and for plane Couette flow. J. Fluid. Mech, 9:371-389, 1960.

[36] M. Westerburg and F. H. Busse. Finite amplitude convection in the presence of finitely conducting boundaries. J. Fluid. Mech, 432:351-367, 2001. 